\title{
Rhythmic activities of the brain: Quantifying the high complexity of beta and gamma oscillations during visuomotor tasks
}

Roman Baravalle, Osvaldo A. Rosso, and Fernando Montani

Citation: Chaos 28, 075513 (2018); doi: 10.1063/1.5025187

View online: https://doi.org/10.1063/1.5025187

View Table of Contents: http://aip.scitation.org/toc/cha/28/7

Published by the American Institute of Physics

\section{Articles you may be interested in}

Bandt-Pompe symbolization dynamics for time series with tied values: A data-driven approach

Chaos: An Interdisciplinary Journal of Nonlinear Science 28, 075502 (2018); 10.1063/1.5022021

Bitcoin market route to maturity? Evidence from return fluctuations, temporal correlations and multiscaling effects

Chaos: An Interdisciplinary Journal of Nonlinear Science 28, 071101 (2018); 10.1063/1.5036517

Chaos in small networks of theta neurons

Chaos: An Interdisciplinary Journal of Nonlinear Science 28, 073101 (2018); 10.1063/1.5028515

State space reconstruction of spatially extended systems and of time delayed systems from the time series of a scalar variable

Chaos: An Interdisciplinary Journal of Nonlinear Science 28, 075504 (2018); 10.1063/1.5023485

Collisions of non-explosive dissipative solitons can induce explosions

Chaos: An Interdisciplinary Journal of Nonlinear Science 28, 075508 (2018); 10.1063/1.5023294

Predicting tipping points of dynamical systems during a period-doubling route to chaos

Chaos: An Interdisciplinary Journal of Nonlinear Science 28, 073102 (2018); 10.1063/1.5038801

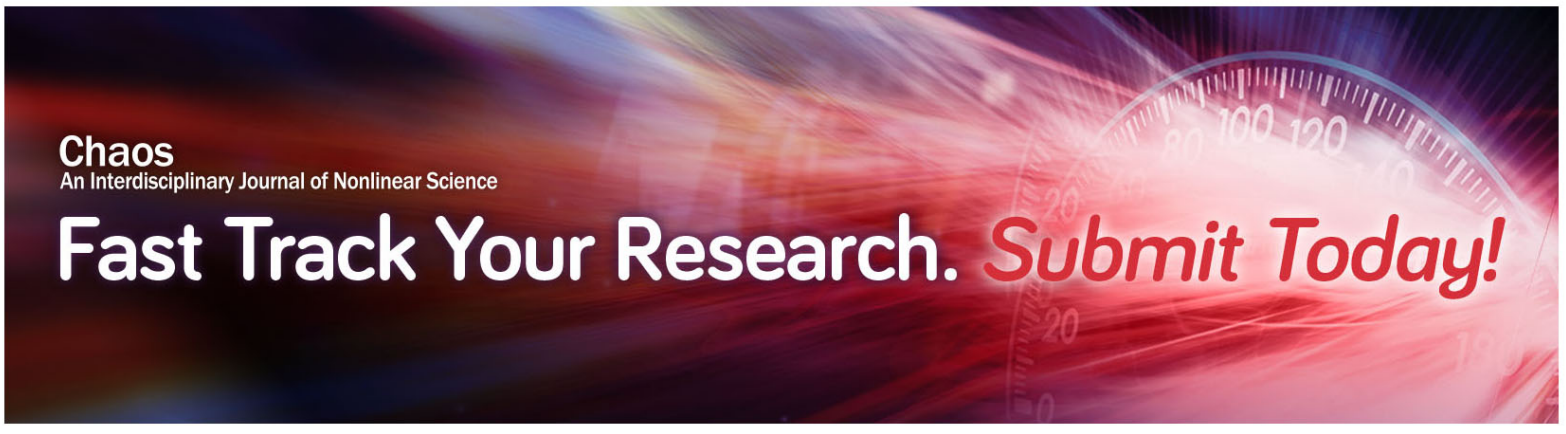




\title{
Rhythmic activities of the brain: Quantifying the high complexity of beta and gamma oscillations during visuomotor tasks
}

\author{
Roman Baravalle, ${ }^{1,2}$ Osvaldo A. Rosso, ${ }^{3,4,5}$ and Fernando Montani ${ }^{1,2, a)}$ \\ ${ }^{1}$ IFLYSIB, CONICET \& Universidad Nacional de La Plata, Calle 59-789, 1900 La Plata, Argentina \\ ${ }^{2}$ Departamento de Física, Facultad de Ciencias Exactas, Universidad Nacional de La Plata, Calle 49 y 115. C.C. 67, 1900 La \\ Plata, Argentina \\ ${ }^{3}$ Departamento de Informática en Salud, Hospital Italiano de Buenos Aires \& CONICET, C1199ABB Ciudad Autónoma de Buenos \\ Aires, Argentina \\ ${ }^{4}$ Instituto de Física,Universidade Federal de Alagoas (UFAL), BR 104 Norte km 97, 57072-970 Maceió, Brazil \\ ${ }^{5}$ Complex Systems Group, Facultad de Ingeniería y Ciencias Aplicadas,Universidad de los Andes, Avenida Monseor Álvaro del \\ Portillo 12.455, Las Condes, Santiago, Chile
}

(Received 07 February 2018; accepted 11 June 2018; published online 20 July 2018)

\begin{abstract}
Electroencephalography (EEG) signals depict the electrical activity that takes place at the surface of the brain and provide an important tool for understanding a variety of cognitive processes. The EEG is the product of synchronized activity of the brain, and variations in EEG oscillations patterns reflect the underlying changes in neuronal synchrony. Our aim is to characterize the complexity of the EEG rhythmic oscillations bands when the subjects perform a visuomotor or imagined cognitive tasks (imagined movement), providing a causal mapping of the dynamical rhythmic activities of the brain as a measure of attentional investment. We estimate the intrinsic correlational structure of the signals within the causality entropy-complexity plane $H \times C$, where the enhanced complexity in the gamma 1, gamma 2, and beta 1 bands allows us to distinguish motor-visual memory tasks from control conditions. We identify the dynamics of the gamma 1, gamma 2, and beta 1 rhythmic oscillations within the zone of a chaotic dissipative behavior, whereas in contrast the beta 2 band shows a much higher level of entropy and a significant low level of complexity that correspond to a non-invertible cubic map. Our findings enhance the importance of the gamma band during attention in perceptual feature binding during the visuomotor/imagery tasks. Published by AIP Publishing. https://doi.org/10.1063/1.5025187
\end{abstract}

EEG is designed to record the electrical activity generated by the brain from electrodes placed on the scalp surface. In this paper, we provide a quantification of the degree of complexity of different brain rhythmic oscillations of the EEG signals by means of information theoretical approach. Our results enhance the functional role of gamma oscillations in the formation of neural representations of perception during visuomotor action and imagery activities.

\section{INTRODUCTION}

Complex systems are highly composite ones that are far from the "perfect order" (e.g., regular crystal) and "complete disorder" (e.g., ideal gas). Indeed, complex systems are usually identified by a certain degree of organization, structure, memory, regularity, symmetry, and intricate patterns. Such systems are therefore not deterministic or random systems, and their complexity is characterized by signatures of spatial and/or temporal correlational structures. A complex system is made up of a very large number of mutually interacting elements, with different kinds of interactions between the components, the temporal patterns, and the dynamics of the system that emerge from those interactions. Therefore, we can identify that the brain is a complex system in which the

\footnotetext{
a)Electronic mail: fmontani@gmail.com
}

dynamical features of neural population activity emerge from the interactions of the neuro-anatomical networks. One of the most important and unsolved questions in neuroscience is how the brain functions relating to the mind. However, understanding how the brain functions pertain to the mind requires characterizing the complex dynamical structure of the neural populations, their organization, and their role into cognitive tasks. The human brain corresponds to a complex system on multiple scales of time and space that has numerous subcomponents with many interactions between them. That is, systems such as the human brain shows a nontrivial component-to-component relationship.

Membrane currents generated by neurons pass through the extracellular space. These currents can be measured by electrodes placed outside the neurons. The field potential (i.e., local mean field) recorded at any given site reflects the linear sum of numerous overlapping fields generated by current sources and sinks distributed along multiple cells. This macroscopic state variable is referred as local field potential (LFP) if measured by a small electrode in the brain. Electroencephalography (EEG) is a technique that allows us to record the electrical activity in different parts of the brain. ${ }^{1}$ The electrical activity of the brain can be considered chaotic and ruled by a nonlinear dynamics. ${ }^{2}$ The EEG is a non-invasive technique recorded on the scalp that often has a poor relationship to the spiking activity of individual neurons. However, the EEG recorded by a single electrode is a 
spatially smoothed version of the local field potentials under a scalp surface on the order of $10 \mathrm{~cm}^{2}$. Therefore, mental states would emerge from the dynamical interaction between multiple physical and functional levels. Distinct brain activity patterns are associated with attentional capture. Neural oscillatory activity patterns are rhythmic neural activities in the brain that can be generated by interactions between neurons. Measures of the relative contribution of EEG oscillations are particularly useful to investigate the emergent properties of the rhythmic activities of the brain. The EEG records the electrical activity of the brain; sensory stimulation or motor output shows different oscillations bands including theta $(\in[4,8)$ $\mathrm{Hz})$, alpha $(\in[8,13) \mathrm{Hz})$, beta $(\in[13,31) \mathrm{Hz})$, and gamma ranges $(\geq 31 \mathrm{~Hz})$. The different rhythms of the brain activity are of functional importance to understand how information is processed in the mammalian brain. In fact, a number of studies have demonstrated that the dynamical changes of the alpha/beta rhythm are associated with normal motor/sensory function. Many studies have shown using EEG ${ }^{3-9}$ or intracranial electroencephalography $(\mathrm{ECoG})^{10-13}$ that humans can use motor imagery to modulate activity in the theta, beta, or gamma bands and to thereby control a brain computer interphase (BCI) system. Interestingly, a subject can change these rhythms without engaging in actual movements, and thus these rhythms could serve as the basis for a BCI. The EEG responses show different spatial and temporal organizations, with distinct responses to modulations of cognitive tasks as they allow us to observe various neural mechanisms and functions. ${ }^{14,15}$ There are indications that gamma activity reflects specific details of movement and increases with motor tasks. ${ }^{16,17}$ Indeed, recent studies have shown relationships of gamma activity with specific kinematic parameters of hand movements. ${ }^{18-21}$ Moreover, it has been previously pointed out in human intracranial EEG that several perceptual, motor and cognitive processes are accompanied by focal energy increases in the gamma band as motor programming, 14,22,23 memory, ${ }^{24,25}$ or visual perception. ${ }^{25,26}$ These findings emphasize the importance of the gamma band when engaging in cognitive activities.

Measurements of entropy- and information-like quantities in spike trains allow us to investigate how the neural code responds to sensorimotor inputs. ${ }^{27-30}$ To estimate these quantities we need to know the distribution of responses, but the number of samples from the distribution is limited by the number of times that the experiment can be repeated inducing therefore sample size dependent bias. Nemenman, Shafee, and Bialek have proposed the NSB method to remove sample dependent bias from entropy-like estimations and from its source, ${ }^{31,32}$ which has been successfully applied to neuronal systems (such as spike singleand multi-unit recordings). ${ }^{27,29,31,32}$ The NSB estimator gave the most reliable estimates of entropy- and information-like quantities. ${ }^{27-32}$ However, the NSB method has not yet been applied to EEG data, where also a limited sample of data is provided by the neurophysiological experiments, and the problem with the estimation of the entropy-like quantities is that they also depend on the recorded distribution of responses. In this paper, we investigate the hypothesis that neural processes associated with visuomotor integration or imaginary task are related to a higher amount of complexity in certain frequency bands. In order to do so, we use the BandtPompe (BP) permutation methodology for the evaluation of the probability distribution function (PDF) ${ }^{33}$ associated with the EEG time series considering the different rhythmic oscillations bands. Based on the quantification of the ordinal "structures" present in the EEG signals and their local influence on the associated probability distribution, we incorporate the time series' own temporal causality through an algorithm of easy implementation and computation. More specifically, we propose an efficient methodology to quantify the degree of complexity within the different oscillations bands of the electrical activity of the brain recorded through the EEG signals while performing a visuomotor or imaginative cognitive task using the BCI2000 system. ${ }^{21,34}$ We precisely quantify the different features of oscillatory patterns considering subtle measures accounting for the causal information: Shannon permutation entropy ${ }^{2,35-39}$ and Martín, Plastino, and Rosso (MPR) permutation statistical complexity. ${ }^{2,35-39}$ Importantly, we used the NSB methodology to remove sample size dependent bias from the entropy and complexity estimations $\mathrm{s}^{32}$ that are estimated using the BP methodology. Our approach allows us to classify the "clustering properties" of the EEG frequency bands, quantifying the causality of the signal and inferring the emergent dynamical of the different oscillation patterns of the brain while performing different tasks of visual-motor or imagery characteristics.

\section{RESULTS}

\section{A. EEG signals}

Electroencephalography is the physiological method that allows us to measure the electrical activity generated by the brain on the scalp surface, and it is based on the propagation of electric impulses along a nerve fibre when the neuron fires. ${ }^{21,34}$

We have considered the EEG Motor Movement/Imagery Dataset recorded using BCI2000 21,34 instrumentation system available through Physionet. ${ }^{21,34,40-42}$ The experimental setup of the BCI2000 system ${ }^{21,34}$ includes an arrangement of 64 electrodes used to record the electrical activity of the brain through the EEG signals while the subjects perform different tasks of visuomotor or imagery characteristics ${ }^{21,34,41-44}$ (see the Appendix for further details). The electrical signals from the brain are picked up by small electrodes that are placed on the subject's head. The electrodes only record the electrical activity of the brain, and importantly, they do not give out electricity. The electrodes do not pick up the electrical signals from individual neurons, but instead, they record the electrical activity from small areas of the brain. The experimental setup of the BCI2000 system is shown in Fig. 1(a) and consists of an arrangement of different used electrodes. ${ }^{21,34,41-44}$ Subjects were asked to perform different motor/imagery tasks, while EEG signals were recorded from 64 electrodes along the surface of the scalp. Eye blink artifacts were generated by fast movements of the eyelid along the cornea, such as during an eye blink. However, muscular artifacts were carefully checked at the beginning of each recording and verified 


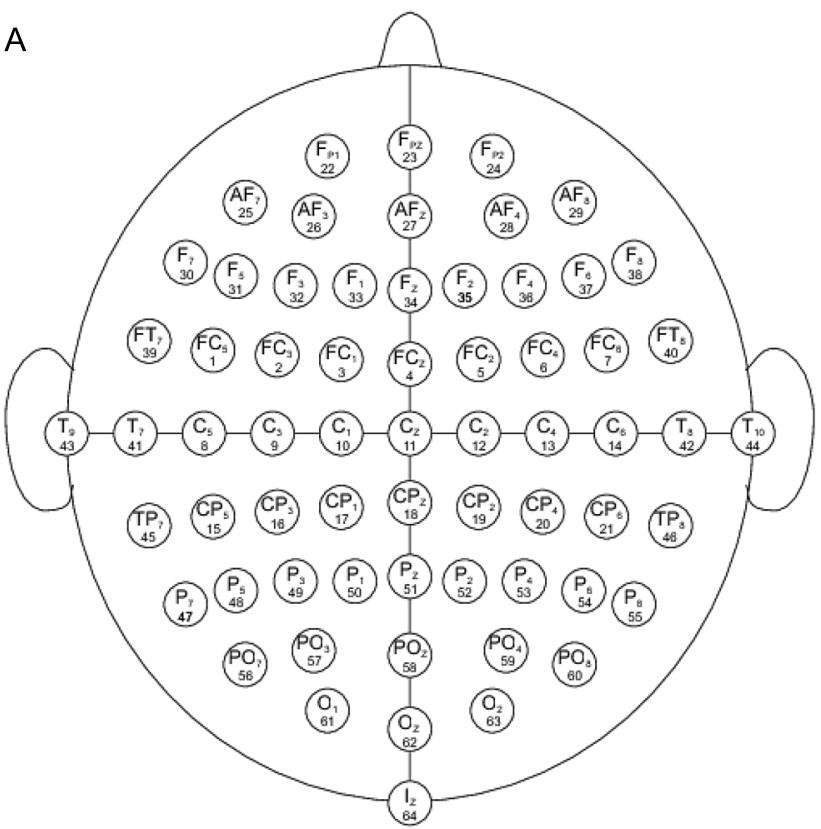

B

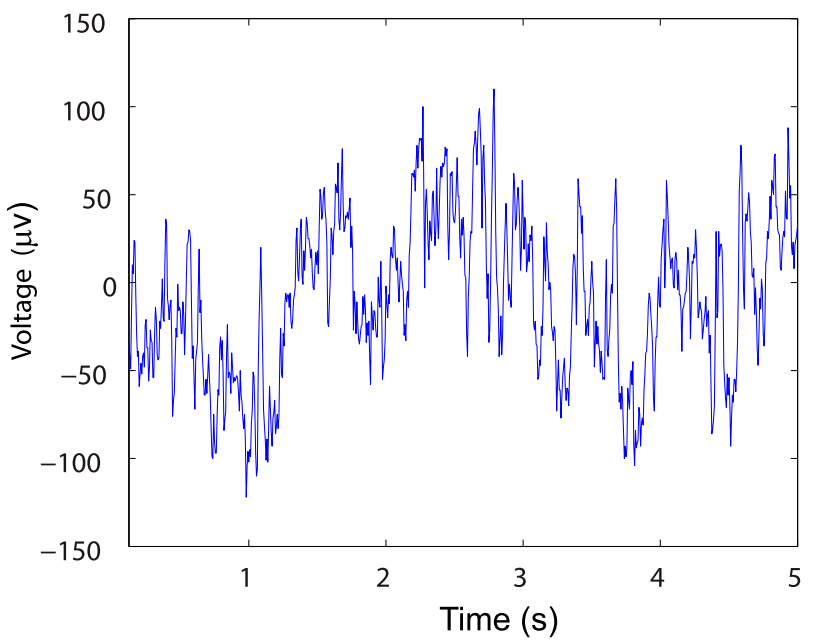

FIG. 1. (a) 64 electrodes used to record the electrical activity of the brain recorded through the EEG signals. (b) A typical raw signal (electrode number 21 - right central parietal).

throughout the recording. ${ }^{21,34,41-44}$ Importantly, in our current analysis, the muscular and technical artifacts of the EEG Motor Movement/Imagery Dataset were removed following the methodology presented in Refs. 21 and 34. That is, a common average reference (CAR) filter is performed before artifact rejection as indicated in Refs. 21 and 34.

A number of oscillatory frequency bands have been linked to different, specific aspects of perceptual processing that are of functional importance to understand how information is processed in the human brain. ${ }^{21,34}$ Figure 1(b) shows a typical raw signal for one of the electrodes presented in Fig. 1(a). Figures 2(a)-2(d) show the filtered signal bands, using the Kaiser filtering window developed by Belitski et al., ${ }^{45}$ of the previous raw signal considering the delta, theta, alpha 1, and alpha 2 bands. Figures 3(a)-3(d) depict the beta 1 , beta 2 , gamma 1 , and gamma 2 oscillation bands of the previous raw signal. For further details of the data processing and oscillation bands, see Sec. 4 in the Appendix.
The EEG bands show brain function and indicate the presence or absence of specific brain activity in specific areas of the brain. However, using the naive sight of bands presented does not reveal new knowledge of how the brain functions related to the mind or which kind of information is carried by the different rhythmic oscillations.

\section{B. Oscillation bands: Visuomotor integration and complexity}

A variety of rhythms in the brain that differ in their frequency are generated by changes on the sensory input and task demands. ${ }^{1,46-49}$ Feedback loops across neurons contribute to the cortical rhythmic activities and modulation of oscillatory phase among neuronal populations, providing deeper insights of how information is processed in the brain. ${ }^{49}$ Our proposal here is to quantify a variety of oscillatory activity patterns of the EEG signals while performing different tasks of motor or imagery characteristics that were recorded using the BCI2000 system. $^{21,34,41-44}$

Many studies have shown using EEG that humans can use motor imagery to modulate rhythmic activity of the bands and to thereby control a BCI system (see Ref. 21 and references therein). We use a versatile method to quantify the complexity of the different oscillation bands of the EEG signals, by means of an information theoretical approach. More specifically, we consider measures accounting for the causal structure of the EEG signals: the Shannon permutation entropy $(H)$ and MPR permutation statistical complexity $(C)$. We refer the reader to Sec. 1 in the Appendix for the definitions of the information quantifiers and theoretical details. We use a filter based on the Kaiser window developed by Belitski et al. ${ }^{45}$ to split the signal in the delta, theta, alpha 1 , alpha 2 , beta 1 , beta 2 , gamma 1 , and gamma 2 oscillation bands (see the Appendix for further details). In order to perform analyses within the BP formalism, we need to consider a large number of points of EEG responses $(M \gg D !)$, we have 20000 data points for each case and we remove bias deviations from the entropy and complexity estimations through the NSB methodology. ${ }^{32}$ This allows us to explain why one should not interpret $H \times C$ results as a consequence of noise artifacts. We used the Bandt and Pompe ${ }^{33}$ methodology for evaluating the PDF, $P$, associated with the time series, considering an embedding dimension $D=6$ and time lag $\tau=1$. This embedding dimension (pattern length) is enough to efficiently capture the information causality of the ordinal structure of the time series. ${ }^{33}$ Confidence error intervals cannot be provided within the BP methodology. As we mentioned previously, the selection of the embedding dimension, $D$, is relevant for obtaining an appropriate probability distribution because $D$ determines not only the number of accessible states (equal to $D$ !) but also the length of the time series, $M$, needed to have a reliable statistics, and therefore, the requirement is that the condition $M \gg D$ ! must be satisfied. Importantly, we estimated the entropy and complexity removing sample size dependent bias from its source through the NSB methodology. ${ }^{32}$ That is, we use the BP method in combination with the NSB algorithm that guarantees estimations of 

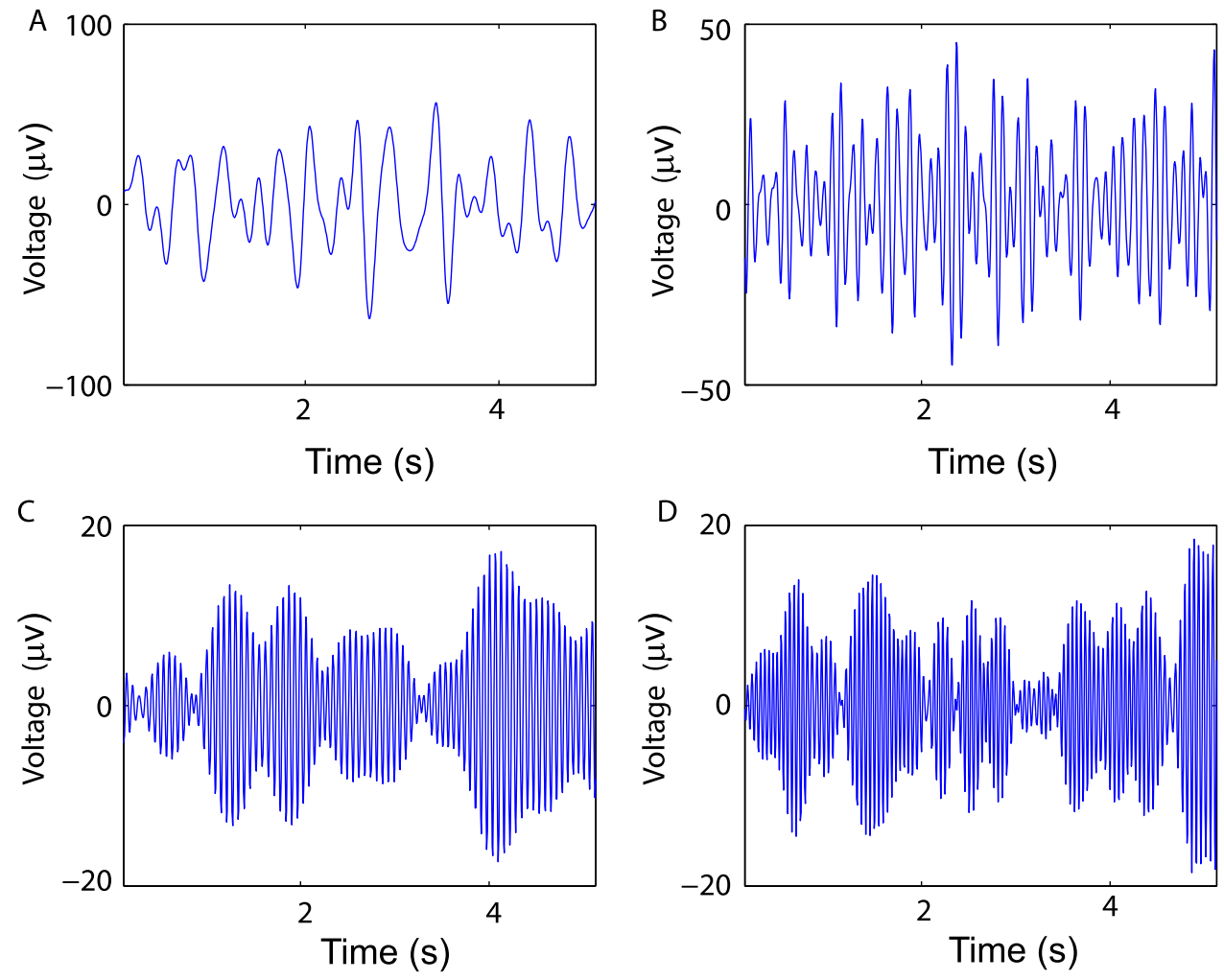

FIG. 2. Filtered signal bands. (a) Delta oscillation band $\in[1,4) \mathrm{Hz}$. (b) Theta oscillation band $\in[4,8) \mathrm{Hz}$. (c) Alpha 1 oscillation band $\in[8,10) \mathrm{Hz}$. (d) Alpha 2 oscillation band $\in[10,13) \mathrm{Hz}$.

entropy and complexity free of bias deviations (see Secs. 1 and 2 in the Appendix for further details).

A proper quantification of the brain rhythms could serve as the basis for a successful BCI. In order to do it so, in the following we analyze the localization of the different oscillation bands within the informational causal plane $H \times C$ (see Sec. 1 in the Appendix for the definitions of Shannon entropy and complexity). Figures 4(a), 4(b), 5(a), and 5(b) show the averaged values of the normalized Shannon entropy considering 109 subjects for the 64 channel EEG considering the different oscillation bands: delta, theta, alpha 1, and alpha 2, respectively. Shannon entropy reaches the maximum values within the beta 2 frequency band and the minimum values for the delta oscillation band, respectively. Figures 6(a), 6(b), $7(\mathrm{a})$, and 7(b) depict the normalized Shannon entropy for the same conditions but taking the beta 1 , beta 2 , gamma 1 , and gamma 2 bands, respectively. In addition, we consider the statistical complexity measure that can detect and quantify noise induced order. ${ }^{35,36}$ Figures $8(a), 8(b), 9(a)$, and 9(b) show the estimations of the complexity under the same conditions for the delta, theta, alpha 1 , and alpha 2 bands, respectively. Figures 10(a), 10(b), 11(a), and 11(b) depict the complexity when considering the beta 1 , beta 2 , gamma 1 , and gamma 2 bands, respectively. Notice that the complexity of the gamma 1 and beta 1 oscillation bands is prevalent, while the complexity of the beta 2 and delta bands is significantly curtailed. Our results are the same for the four different visuomotor/imagined tasks.

Finally, Figs. 12(a) and 12(b) show the normalized Shannon entropy and the complexity for the whole signal, respectively. Let us remark that we name as whole signal to the raw one without considering any filtering. The averaged values of the entropy are much higher than those depicted in the case of the different oscillation bands and are very close to a random state. Furthermore, in this case, the complexity results to be much lower than when considering the different oscillation bands.

Ultimately, Fig. 13 shows the informational causal plane of entropy versus complexity, $H \times C$, averaging over the 64 channels and considering all the subjects, taking into account the different frequency bands. The complexity depends on the structure of the brain and we observed some variations in the $H \times C$ plane for the gamma 1 point, depending on the region of the brain in which the electrodes are located. However, the gamma 1 point remains always as a maximum in $H \times C$ plane for the different electrodes, and therefore in Fig. 13 we present the averaged values. That is, Fig. 13 depicts the global behavior of the entropy-complexity oscillation bands across the brain. The current analysis shows the results that are coincident for the four different visual-motor/imaginary tasks (specified within the Appendix). Let us remark that the MPR statistical complexity reaches a maximum at the gamma 1 band, and the beta 1 rhythm depicts a slightly lower value. Let us emphasize that the gamma 1 and beta 1 oscillation bands are not in order or disorder state as the Shannon entropy is non zero or maximal but are instead "in between" in a chaotic state of the brain. It is important to remark that we use as control signal the shuffled baseline signal that as expected shows the maximal disorder or a maximal value of entropy but with zero complexity. The delta band depicts a low Shannon entropy value, and therefore, the system is in a very ordered 
A

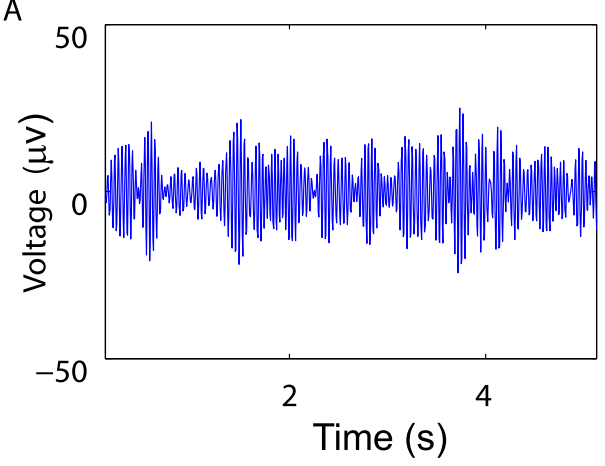

C

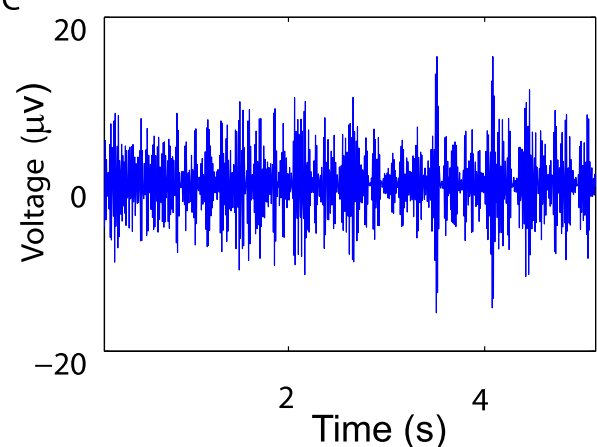

B
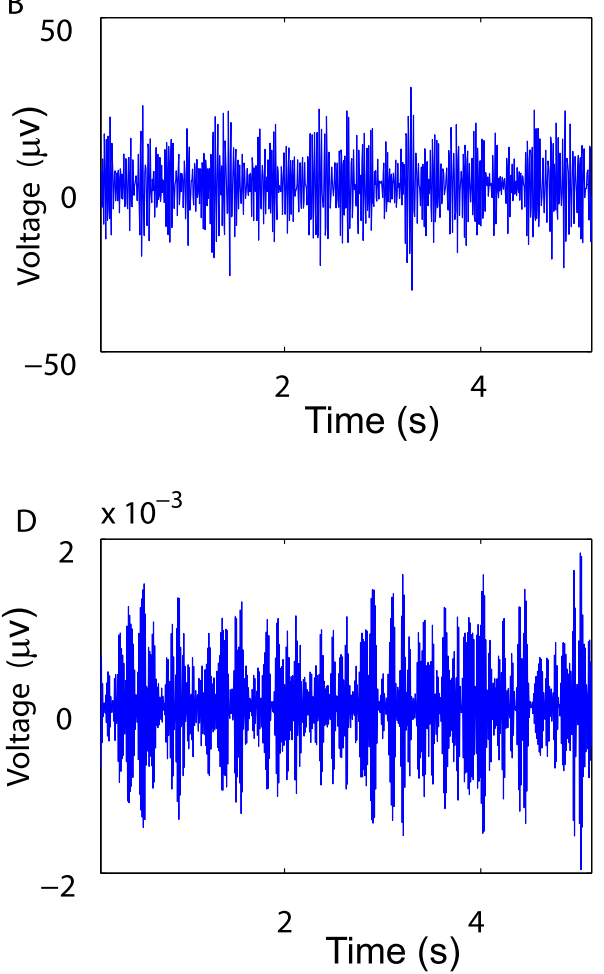

FIG. 3. Filtered signal bands. (a) Beta 1 oscillation band $\in[13,18) \mathrm{Hz}$. (b) Beta 2 oscillation band $\in[18,31) \mathrm{Hz}$. (c) Gamma 1 oscillation band $\in[31,41) \mathrm{Hz}$. (d) Gamma 2 oscillation band $\in[41,50) \mathrm{Hz}$. state which means that it is represented by a very narrow probability distribution (normalized Shannon entropy closer to zero). In contrast, the system is in a very disordered state when considering the beta 2 oscillation band that corresponds to an almost flat probability distribution (normalized Shannon entropy close to the maximal value). Importantly, the values for the whole signal are very close to a random distributed state. This is the case even when filtering the signal between 1 and $50 \mathrm{~Hz}$ due to the impedance of the 64 electrodes used for the recording (see the Appendix).

The continuous lines represent the curves of maximum and minimum statistical complexity, $C_{\max }$ and $C_{\min }$, respectively, as functions of the normalized Shannon entropy ${ }^{50}$ (values that depend only on the number of degree of freedom of the considered PDF). Note that the maximum $C_{\max }$ and the minimum $C_{\min }$ values of the complexity restrict the possible values of the intensive statistical complexity in the $H \times C$ plane. Localization in the entropy-complexity plane $H \times C$, in some cases, closely approaches the limiting curve of maximum statistical complexity $C_{\max }$. However, the evaluation of higher values of the complexity provides additional insight into the details of the system's probability distribution, which is not discriminated by randomness measures like the entropy. ${ }^{51,52}$ Complexity helps to uncover information related to the correlational structure of the physical process under study. The entropy-complexity diagram (or plane), $H \times C$, allows us to detect subtle changes in the neural dynamics of a system originated by modifications of the external stimuli. This provides a distinction between chaotic-deterministic and stochastic dynamics. ${ }^{2,35,36,38,39,53}$ Note that neural oscillatory activity patterns are rhythmic neural activities in the brain that can be generated by interactions between neurons. Particularly, beta rhythm changes are associated with the normal motor/sensory function. In addition, oscillatory activity within the gamma 1 shows the very important role of the very high frequency in the timing and integration process of neuronal networks in the brain that may serve to assess the temporal dynamics of cortical networks and their interactions.

\section{DISCUSSION}

Cognitive neuroscience partially relies on the techniques to map the neural networks underlying cognition in humans such as EEG recordings. The EEG accounts for the electrical activity oriented perpendicular to the surface of the brain and records fluctuations in the neural electric fields. The brain is made up of millions of neurons. Neurons control the body's functions by communicating using electrical signals. Neurons send nerve impulses from one cell to another to transfer messages around the brain. These messages, called action potentials, are due to changes in the electrical charge of the neuronal cells. That is, neuronal cells communicate using electrical signals, and when they do this they "give off" electricity staying quiescent. At population level, most neurons are quiescent but can fire spikes when properly stimulated, and they typically respond by producing complex spike sequences. This effect provides insights of the intrinsic dynamics of the neurons and in some extent of the temporal characteristics of the stimulus. It is the electrical activity, sometimes called "brain oscillations", that are picked up on by EEG. Particularly, the significance of rhythmic patterns for information processing has been pointed out by Kayser et al. ${ }^{54}$ Oscillations can modulate information processing as rhythmic inhibition plays an important role in oscillations throughout the brain eliciting rebound bursts and resulting in re-excitation of neurons, regulating the neuronal firing in the brain. ${ }^{53,55}$ Understanding 

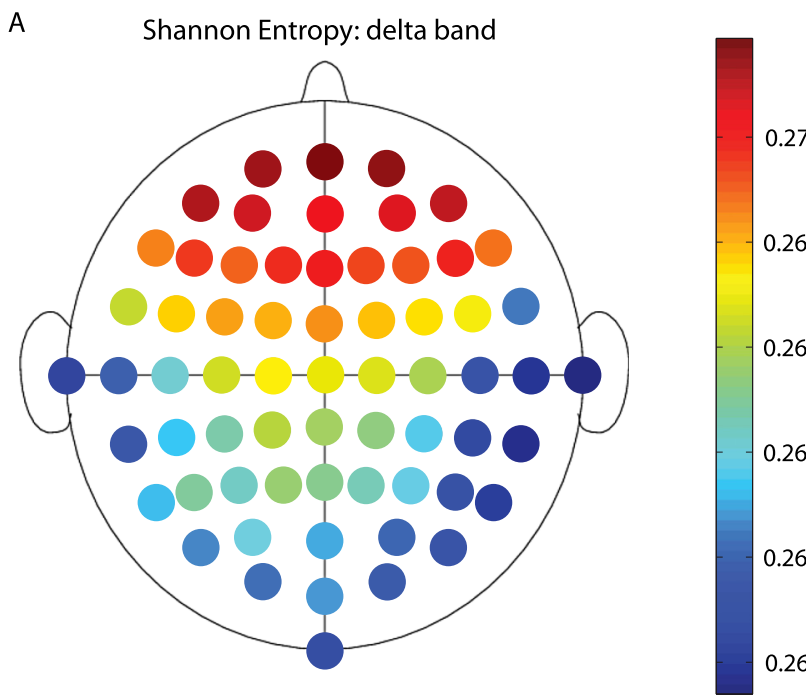

B
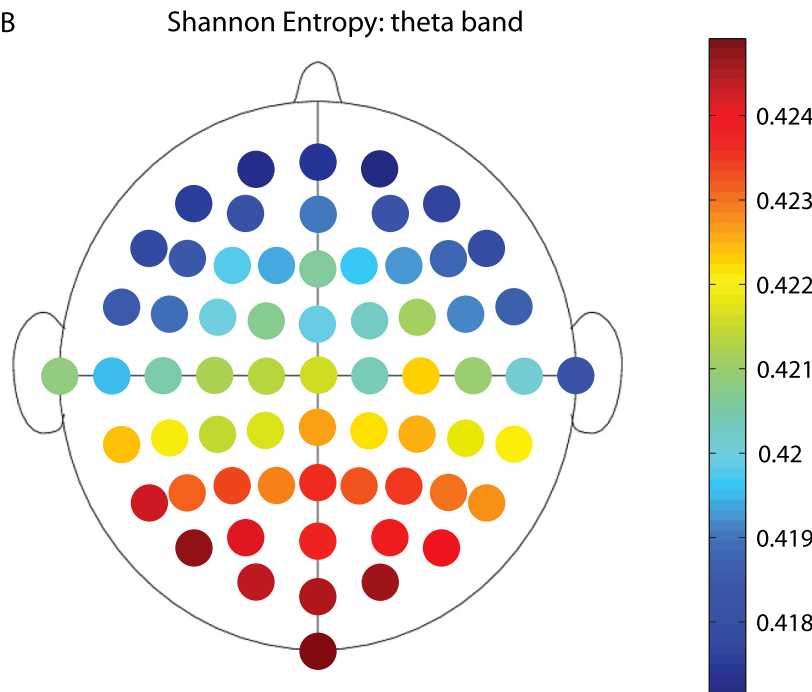

FIG. 4. Averaged values of the normalized Shannon entropy considering 109 subjects for the 64 channels of the EEG. (a) corresponds to the delta band and (b) to the theta oscillation band. We consider $D=6$ and $\tau=1$. Delta oscillation band corresponds to $\in[1,4) \mathrm{Hz}$ and theta oscillation band to $\in[4,8) \mathrm{Hz}$.

the features of neuronal responses encoding the variations in the stimuli is an important challenge in neuroscience. Oscillations in the brain may be generated by non-invasive brain stimulation, either by intrinsic mechanisms or by interactions between them, ${ }^{1,46-49}$ and may have a crucial role in feature binding, information transmission, and the generation of rhythmic motor output even in isolation from motor and sensory feedback. ${ }^{56}$ They are indeed manifested as a variety of rhythms that differ in their frequency, origin, and reactivity to changes in sensory input and external stimuli. ${ }^{1,46-49}$

The statistical complexity is a measure derived from information theory that allows us to quantify the global dynamics of the electrocortical activity. The measure of statistical complexity permits us to quantify critical details regarding the dynamic processes underlying the distribution. The perfect order (for example, a periodic sequence) and the maximum randomness (a fair coin toss) can be described very easily because they do not have any structure. That is, in

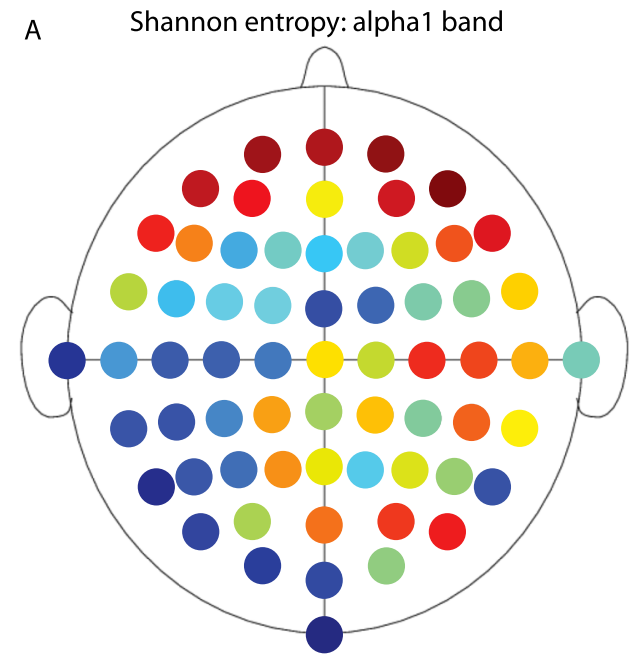

0.4632

0.463

0.4628

0.4626

0.4624

0.4622

0.462

0.4618

0.4616

0.4614

B Shannon entropy: alpha2 band

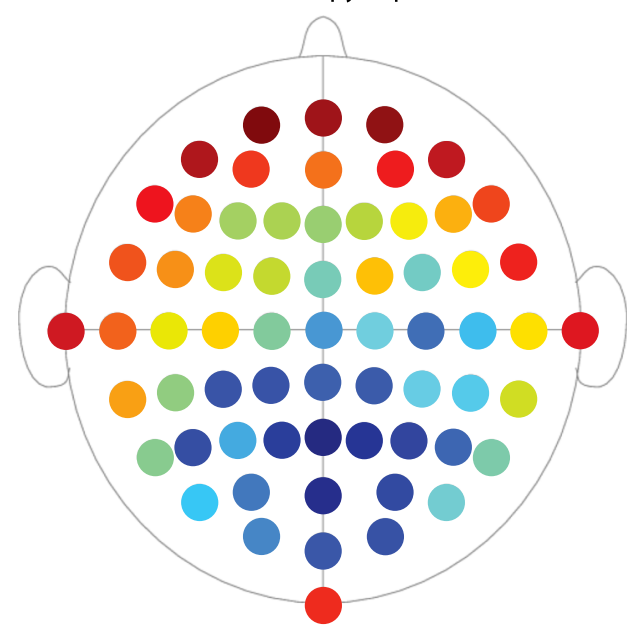

0.5015

0.501

0.5005

$-0.5$

0.4995

0.499

0.4985

FIG. 5. Averaged values of the normalized Shannon entropy considering 109 subjects for the 64 channels of the EEG. (a) corresponds to the alpha 1 band and (b) to the alpha 2 oscillation band. We consider $D=6$ and $\tau=1$. Alpha 1 oscillation band corresponds to $\in[8,10) \mathrm{Hz}$ and to alpha 2 oscillation band $\in[10,13) \mathrm{Hz}$.

these both cases, the statistical complexity is zero. However, between these two extremes, there is a wide range of ordinal structures. The nonlinear dynamics of the brain is of dissipative nature, and subject to strong non-equilibrium conditions that characterize the emergent properties of the neurons at large scale and delineate the complex behavior of the neuronal functions. Chaos was first described by Poincare in the 1800s, and recent years have witnessed major developments in the physics of brain systems. ${ }^{57-59}$ We have investigated the chaos characteristics of EEG oscillations patterns and its relation with the complex structure of the neuronal networks. Our results show that the beta 1 and gamma bands are both in the chaotic attractors zone, corresponding to the localization of a dissipative chaotic system. As can be appreciated from the contrast of our results with the one being shown in Ref. 60, in which the Bandt-Pompe PDF was evaluated considering $D=6$ (pattern length) and $\tau=1$ (time lag), our results correspond to the localization of a chaotic dissipative system similar to the Tinkerbell map X-component. ${ }^{60}$ On the 
A Shannon entropy: beta1 band
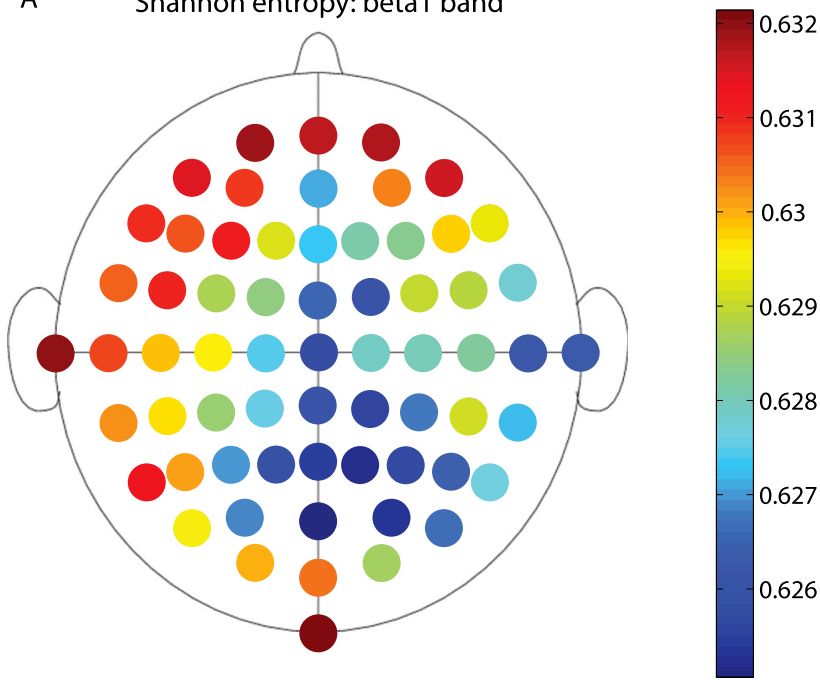

B Shannon entropy: beta2 band
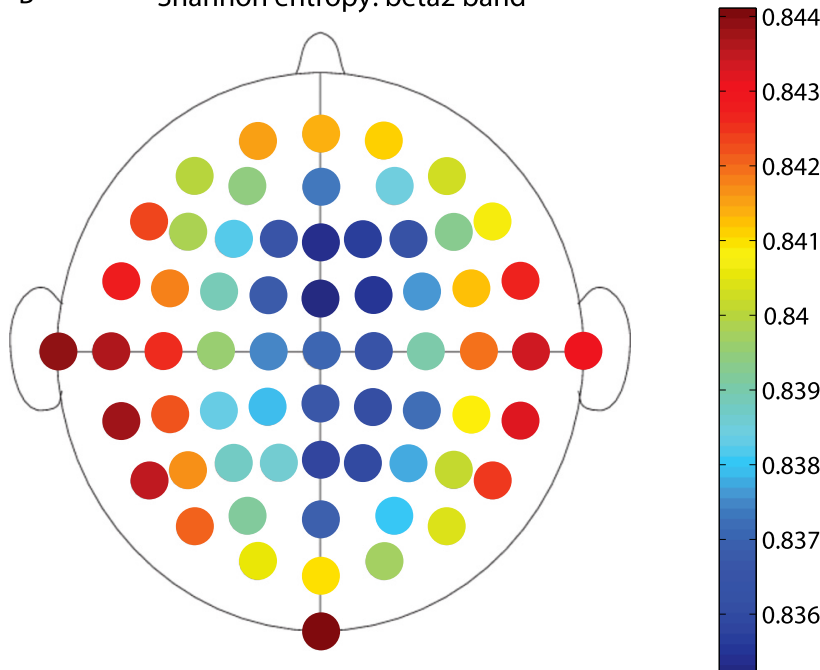

FIG. 6. Averaged values of the normalized Shannon entropy considering 109 subjects for the 64 channels of the EEG. (a) corresponds to the beta 1 band and (b) to the beta 2 oscillation band. We consider $D=6$ and $\tau=1$. Beta 1 oscillation band corresponds to $\in[13,18) \mathrm{Hz}$ and beta 2 oscillation band to $\in[18,31) \mathrm{Hz}$.

other hand, the control randomized signal shows zero complexity and complete disorder. The higher complexity values in the alpha band support the finding that these frequencies process aspects of attention. ${ }^{61}$

Thus, complexity provides important additional information regarding the peculiarities of the underlying PDF, that is not detected by the entropy. Notice that is the combination of both entropy and complexity depicted the plane, $H \times C$, that allow us to distinguish the different rhythmic oscillations bands. In other words, entropy and complexity are different concepts that are complementary to each other. Summarizing, the system's localization in an entropy-complexity plane $(H \times C)$ provides a global quantifier that displays typical specific features associated with its dynamics' nature of the brain. Thus, it is clear that important additional information related to the correlational structure of the different oscillation bands is provided by evaluating the statistical complexity measure. Our approach allows us to characterize the dynamics of EEG
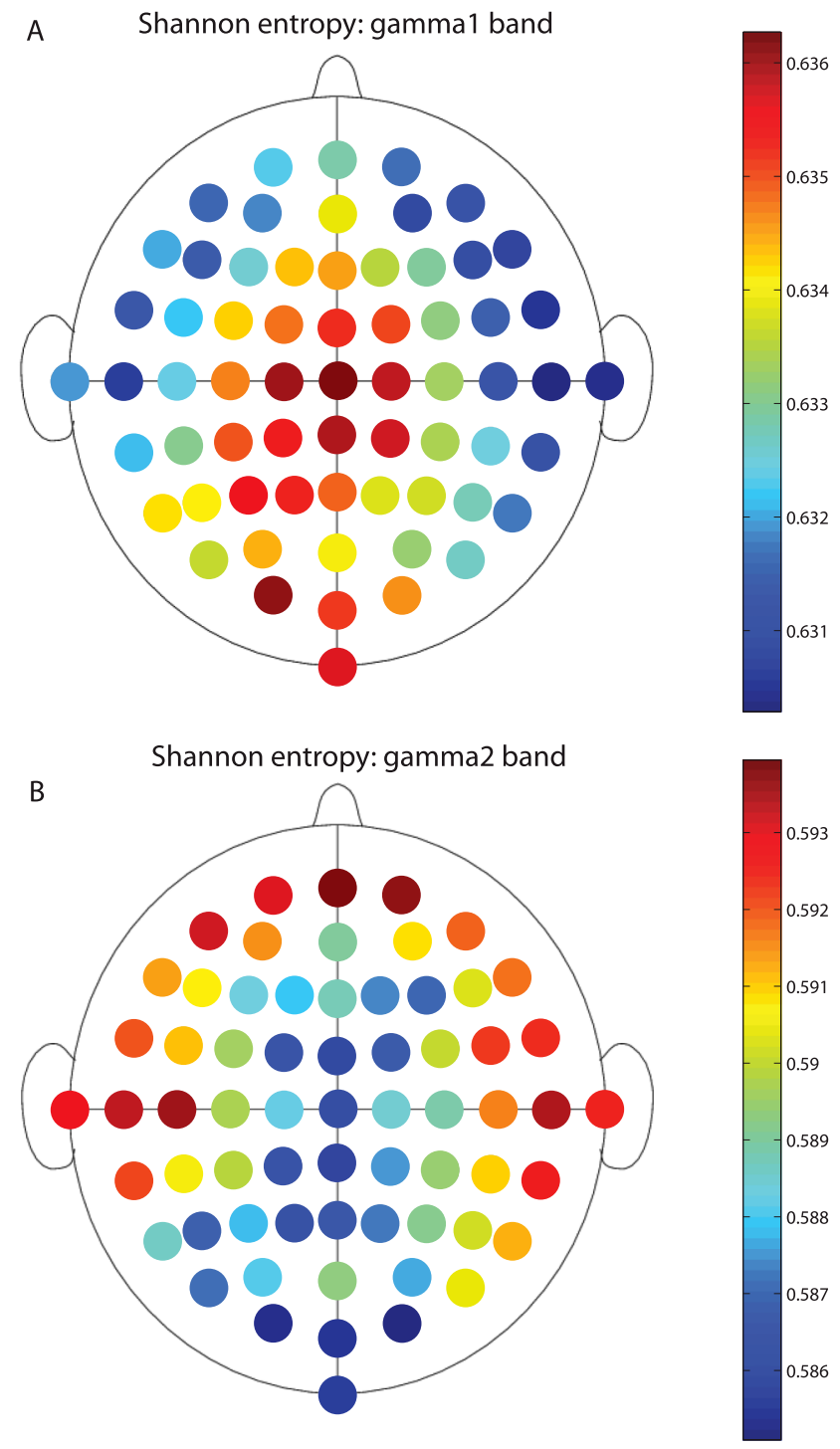

FIG. 7. Averaged values of the normalized Shannon entropy considering 109 subjects for the 64 channels of the EEG. (a) corresponds to the gamma 1 band and (b) to the gamma 2 oscillation band. We consider $D=6$ and $\tau=1$. Gamma 1 oscillation band corresponds to $\in[31,41) \mathrm{Hz}$ and gamma 2 oscillation band to $\in[41,50) \mathrm{Hz}$.

signals, quantifying the causality of the signal for the different oscillation bands, and inferring the emergent properties of the system when performing visuomotor/imagery tasks.

The enhanced activity in the gamma and beta 1 bands helps distinguish visual memory from nonmemory control conditions. The gamma-band activity involves higher frequencies in the EEG and has been observed during a variety of behavioral states in human brain studies. Its functional significance is far from being fully understood, however, gamma-band oscillations have been related to cognitive functions such as attention, learning, and memory. Enhanced activity in the gamma and beta bands over occipital and frontal regions distinguished visual memory from nonmemory control conditions. ${ }^{62,63}$ Synchronous oscillatory activities in the beta-gamma bands were hypothesized to code the relationship among visual features. These oscillations are known to be driven by the bottom-up visual inputs in the adult mammalians. Our findings show that alpha rhythmic activity of 

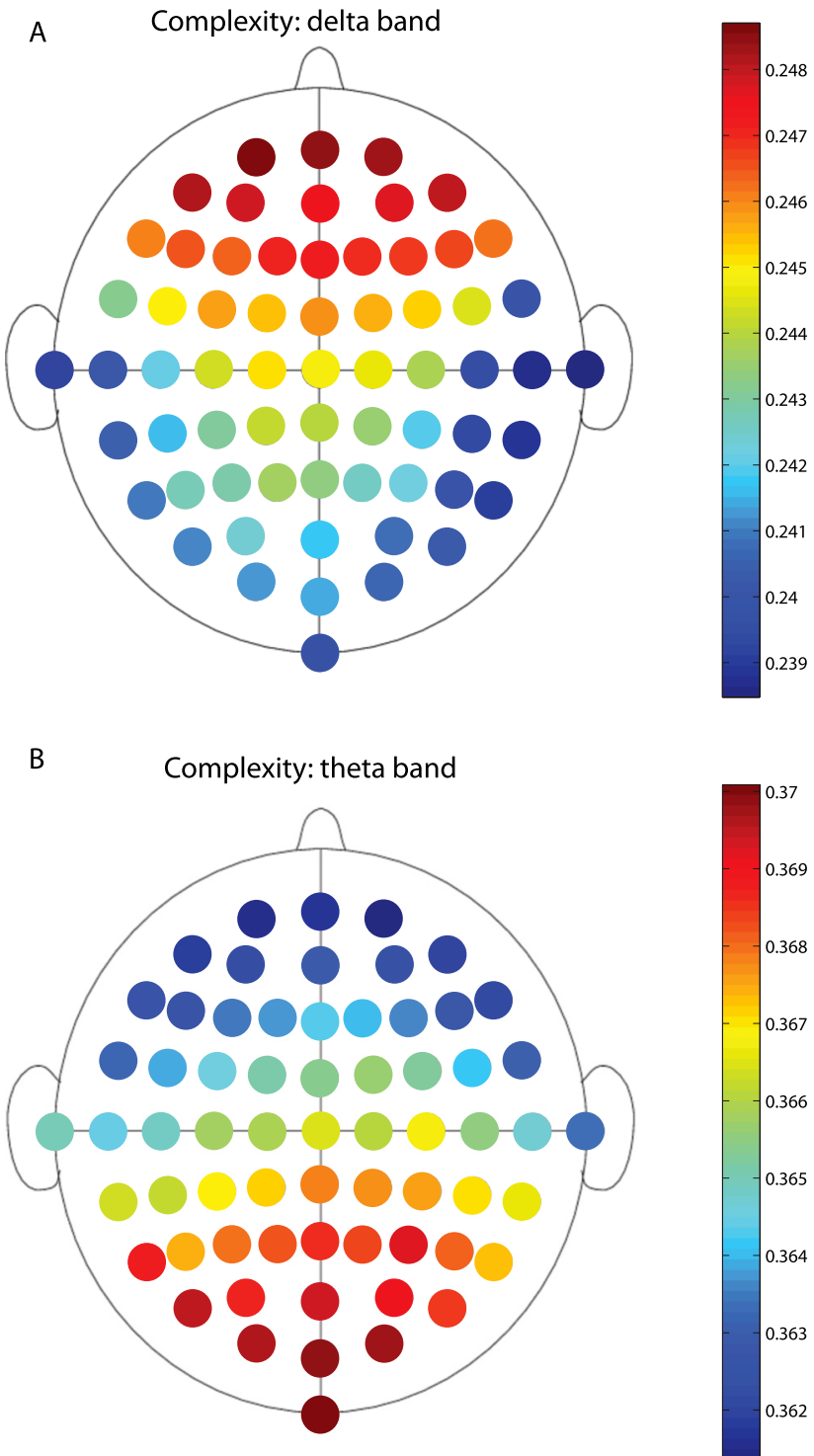

FIG. 8. Averaged values of the complexity considering 109 subjects for the 64 channels of the EEG. (a) corresponds to the delta band and (b) to the theta oscillation band. We consider $D=6$ and $\tau=1$. Delta oscillation band corresponds to $\in[1,4) \mathrm{Hz}$ and theta oscillation band to $\in[4,8) \mathrm{Hz}$.

visuomotor task is closely related with an enhancement of the complexity due to the attention-demanding cognitive processes. This state is accompanied by high complexity values of the beta 1 band and a curtailment of this quantity in the theta band.

Developing new analysis based on complexity measures instead of estimating parameters that show general characteristics of the amplitude and frequency is essential, as it will open new opportunities for understanding the functional significance of brain activity in health and disease. Recent advances in developing novel mathematical approaches for investigating the temporal structure of brain activity based on complexity measures, such as MPR complexity, can provide insights that go beyond those obtained with conventional techniques of signal analysis. The complexity of the brain would represent the amount of "information" contained in the oscillation band, in the sense that it quantifies the dynamical features of the temporal pattern due to functional interactions

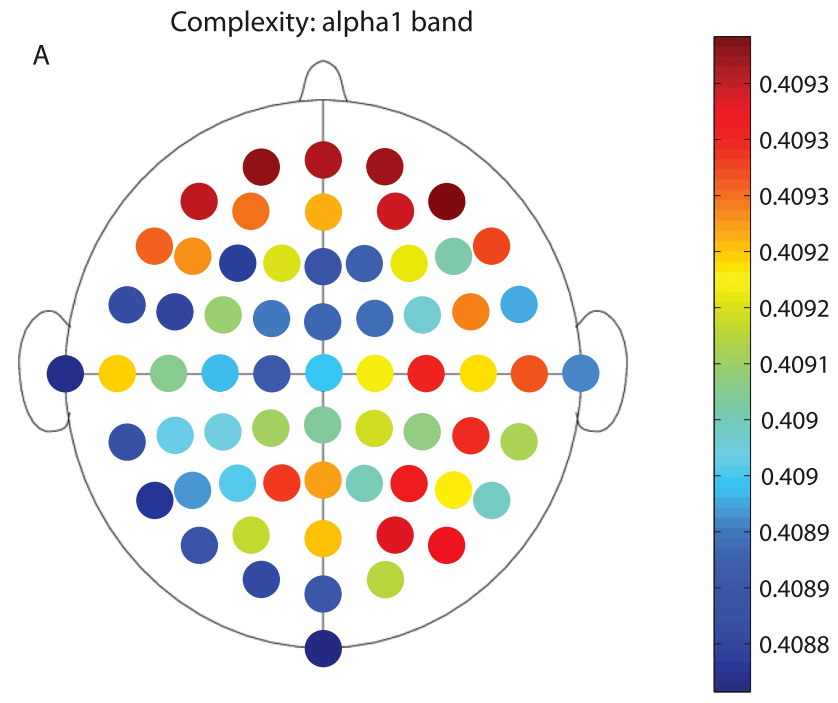

B Complexity: alpha2 band

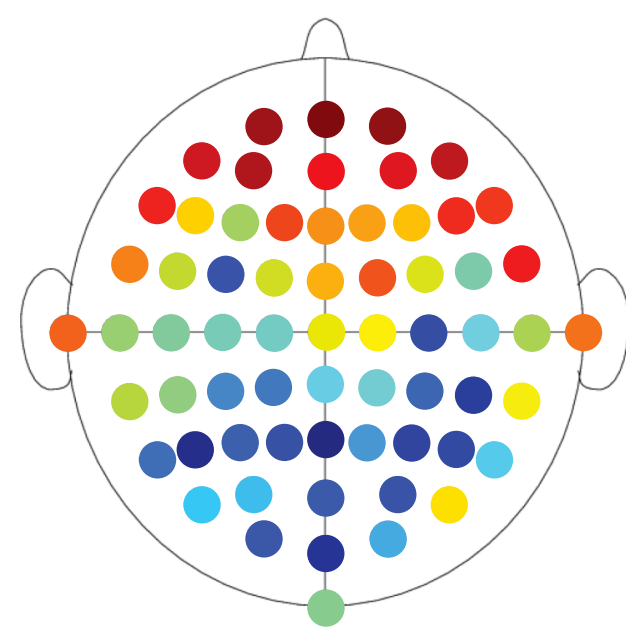

0.4198

0.4196

0.4194

0.4192

0.419

0.4188

FIG. 9. Averaged values of the complexity considering 109 subjects for the 64 channels of the EEG. (a) corresponds to the alpha 1 band and (b) to the alpha 2 oscillation band. We consider $D=6$ and $\tau=1$. Alpha 1 oscillation band corresponds to $\in[8,10) \mathrm{Hz}$ and alpha 2 oscillation band to $\in[10,13) \mathrm{Hz}$.

produced by a structural network. Complexity captures the degree to which a neural system integrates specialized information, and in particular, complexity can distinguish time series generated by stochastic and chaotic systems. ${ }^{35,36}$

The goal of our present work was to identify the brain rhythmic oscillations associated with successful visuomotor integration for a possible future application to BCI systems. Our hypothesis is that neural processes associated with visuomotor integration or imagery task are related to a higher amount of complexity in certain frequency bands. We specifically estimate the causality entropy-complexity plane $H \times C$, showing that the complexity of the electrical activity can be characterized by computing the intrinsic correlational structure of the EEG signal. This allows us to identify the dynamics of the brain within the zone of a chaotic dissipative behavior in the plane $H \times C$. Our findings suggest that the elevated complexity in the gamma 1 , beta 1 , and gamma 2 bands reflects the chaotic nature of EEG activity. 

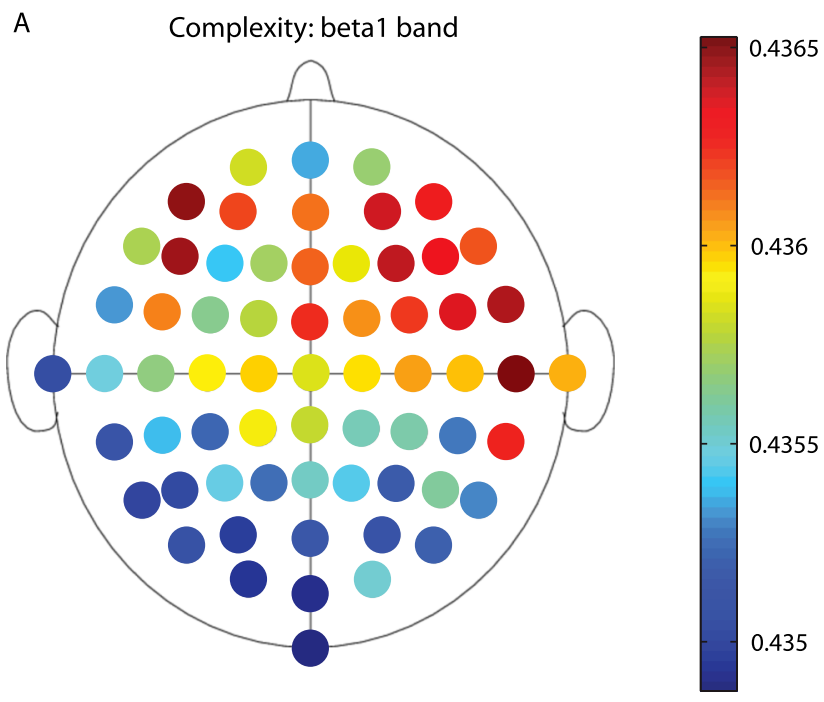

B
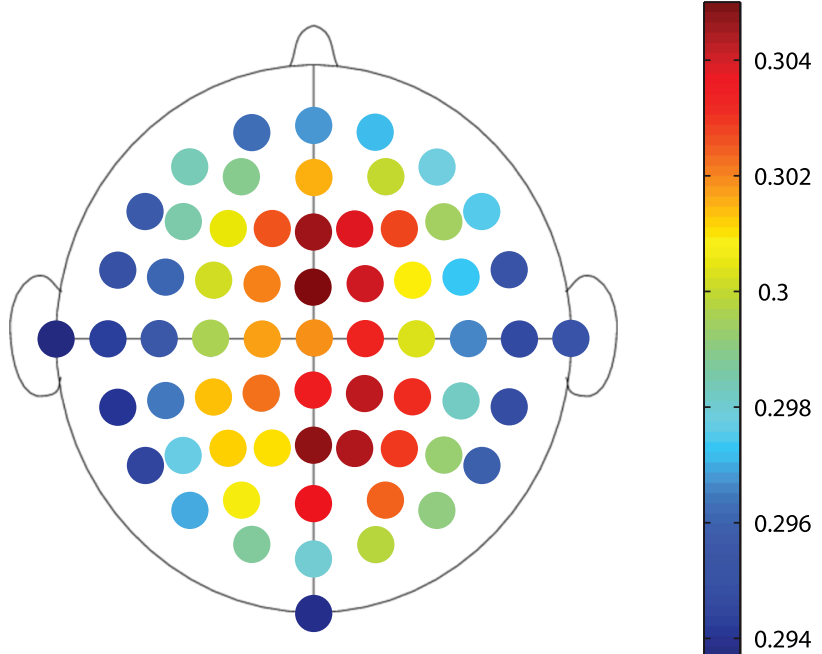

FIG. 10. Averaged values of the complexity considering 109 subjects for the 64 channels of the EEG. (a) corresponds to the beta 1 band and (b) to the beta 2 oscillation band. We consider $D=6$ and $\tau=1$. Beta 1 oscillation band corresponds to $\in[13,18) \mathrm{Hz}$ and beta 2 oscillation band to $\in[18,31) \mathrm{Hz}$.

The elevated complexity during the visuomotor or imagery task arises mostly from the activity of high frequency bands (beta 1, gamma 1, and gamma 2) than from the intermediate frequency bands (theta and alpha), showing that complexity is not just a property of the low frequency components of the spectrum. A proper quantification of the complexity of the brain function, such as cognition, has been one of the major challenges in neuroscience. One main challenge in computational neuroscience is to provide an efficient algorithm of brain-computer interface (BCI) that could translate the recorded neural activity into a control signal for an external device. The signal acquisition component is generally divided into two categories: noninvasive and invasive. The causality entropy-complexity plane $H \times C$ could serve as the basis for a successful BCI, a technology that can help people who are severely disabled connected to a noninvasive EEG device. That is, by identifying the meaningful signal information of the EEG signal within the plane $H \times C$, we can offer a mapping of human cognitive or sensory-motor

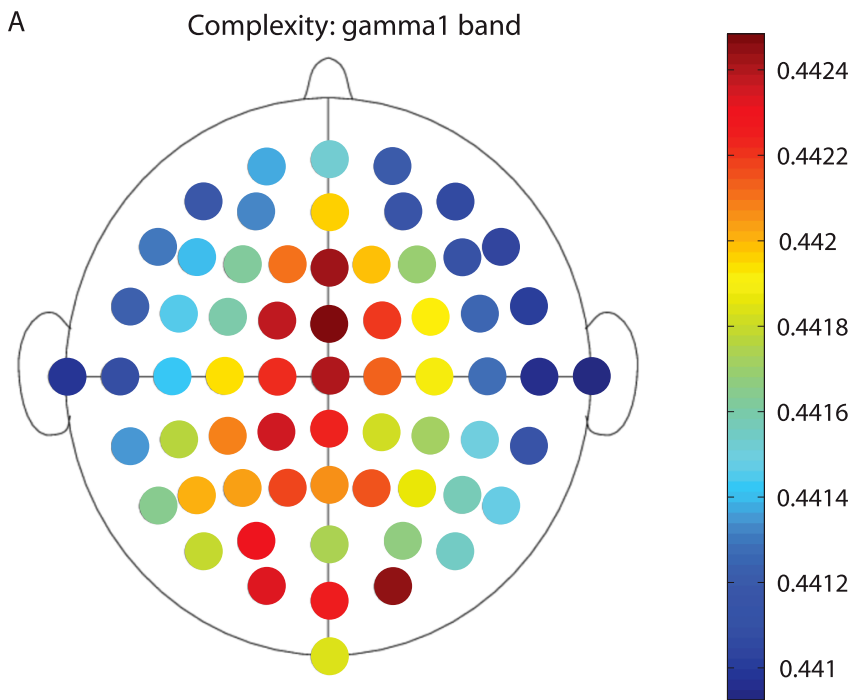

B Complexity: gamma2 band

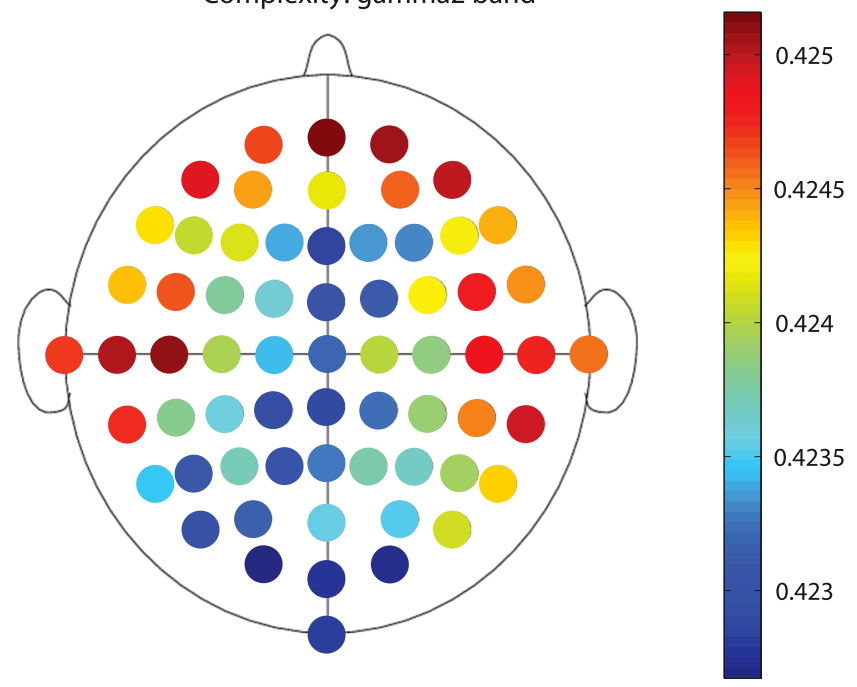

FIG. 11. Averaged values of the complexity considering 109 subjects for the 64 channels of the EEG. (a) corresponds to the gamma 1 band and (b) to the gamma 2 oscillation band. We consider $D=6$ and $\tau=1$. Gamma 1 oscillation band corresponds to $\in[31,41) \mathrm{Hz}$ and gamma 2 oscillation band to $\in[41,50) \mathrm{Hz}$.

functions that could be decoded into certain movements of artificial actuators.

\section{ACKNOWLEDGMENTS}

We gratefully acknowledge PIP 11220130100327CO (2014/2016) CONICET, Argentina (F.M.), and Universidad Nacional de La Plata, Argentina (project 11/X812).

\section{APPENDIX: METHODS}

\section{Shannon entropy and MPR statistical complexity}

Measuring different trails of the same experiment is the most important element for any scientific research. These sequences (or trails) are usually named as time series, from which one must carefully extract information about the dynamical system being tested. Therefore, we need to define an information theory quantifier as a measure capable of characterizing a given property of the probability distribution 
A

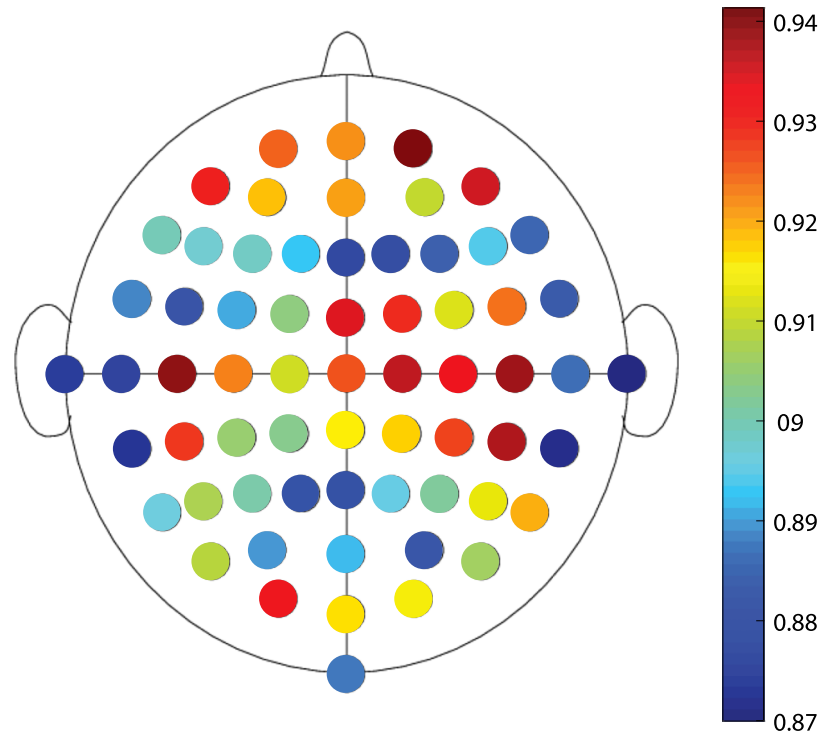

B

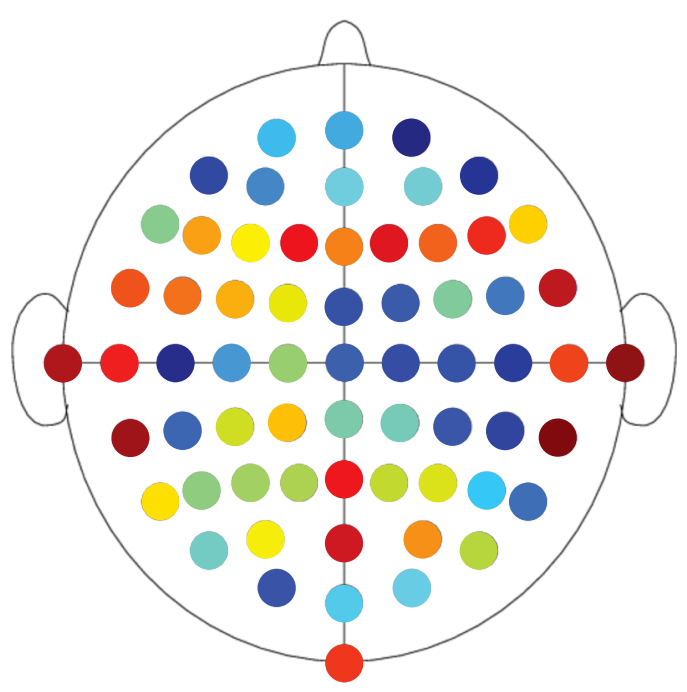

0.94

0.93

0.92

0.91

09

0.89

0.88

0.87

Complexity: whole signal
FIG. 12. Whole signal without any filtering considering 109 subjects for the 64 channels of the EEG. (a) corresponds to the averaged values of the normalized Shannon entropy and (b) to complexity ( $D=6$ and $\tau=1)$.

function (PDF) associated with these time series of the signal (for instance, the EEG signals).

Entropy provides us a measure of uncertainty and is the most archetypal example of the information quantifiers. For a continuous probability distribution function $f(x)$ with $x \in$ $\Delta \subset \mathbb{R}$ and $\int_{\Delta} f(x) d x=1$, we define the Shannon Entropy $S^{64}$ as

$$
S[f]=-\int_{\Delta} f \log _{2}(f) d x .
$$

Given a time series $\mathcal{X}(t) \equiv\left\{x_{t} ; t=1, \ldots, M\right\}$, a set of $M$ measures of the observable $\mathcal{X}$, and the associated PDF, given by $P \equiv\left\{p_{j} ; j=1, \ldots, N\right\}$ with $\sum_{j=1}^{N} p_{j}=1$ and $N$ the number of possible states of the system under study, the Shannon's

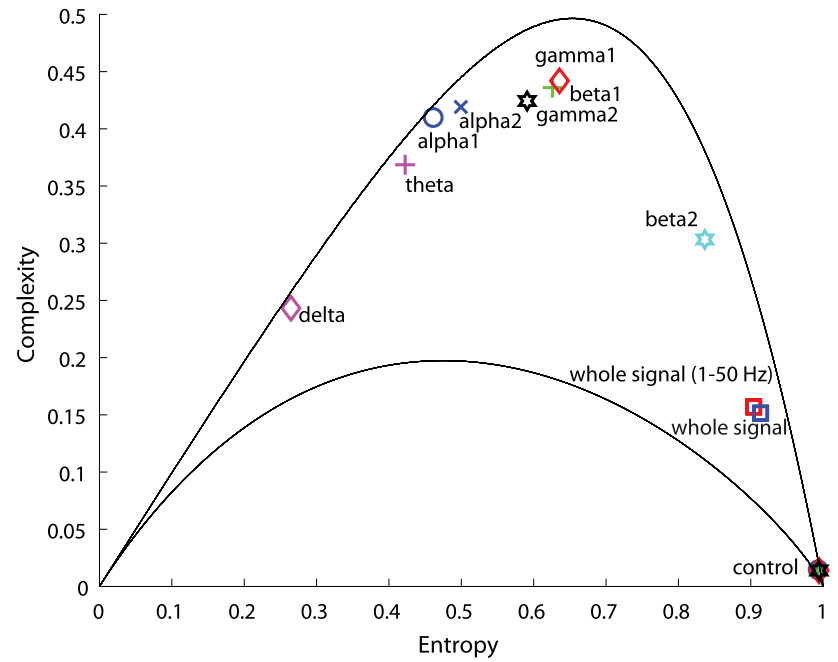

FIG. 13. Causal MPR complexity versus normalized Shannon entropy $(H \times$ $C$ plane), averaging over the 64 channels and considering all the subjects, taking into account the different frequency bands. We consider $D=6$ and $\tau=1$.

logarithmic information measure ${ }^{64}$ is defined by

$$
S[P]=-\sum_{j=1}^{N} p_{j} \log _{2}\left(p_{j}\right) .
$$

This functional is equal to zero when we are able to predict with certainty which of the possible results $j$, whose probabilities are given by $P_{0}=\left\{p_{j^{*}}=1\right.$ and $p_{j}=0$ for all $\left.j^{*} \neq j\right\}$, it will really take place. Thus, the knowledge of the underlying process is maximum in this case. On the contrary, this knowledge is minimal for a uniform distribution $P_{e}=\left\{p_{j}=\right.$ $1 / N \forall j=1, \ldots, N\}$.

The entropy of Shannon $S$ is a measure of the "global character" that is not very perceptive to large variations in the PDF that takes place in a short zone. However, it is important to point out that ordinal structures present in a process are not quantified by randomness measures. That is, measures of statistical or structural complexity are necessary for a better characterization of the dynamics of the system represented by its time series (for further details we refer the reader to Ref. 65. The opposite ends of perfect order (for instance, a periodic sequence or a regular crystal) and maximum randomness (that is, a fair coin toss or an ideal gas). Both cases are trivial to describe because they have no structure and the complexity $C$ should be zero in these cases, with $C\left[P_{0}\right]=$ $C\left[P_{e}\right]=0$, for $P_{0}$ and $P_{e}$, the PDFs of the perfectly ordered system and maximum disordered, respectively. Importantly, it was shown that for a given value of the normalized Shannon entropy $H=S / S_{\max }$, the range of possible values $C$ varies between a minimum $C_{\min }$ and a maximum $C_{\max }$, restricting the possible values of the statistical complexity in a certain entropy complexity plane. ${ }^{50}$

Let us consider the MPR statistical complexity, ${ }^{66} C_{J S}$, that quantifies the accurate details of the dynamics of the system under investigation. This measure is based on the groundbreaking research conducted by López-Ruiz et al. ${ }^{67}$ the statistical complexity measure (SCM) is written as

$$
C_{J S}[P]=\mathcal{Q}_{J}\left[P, P_{e}\right] \cdot H[P],
$$



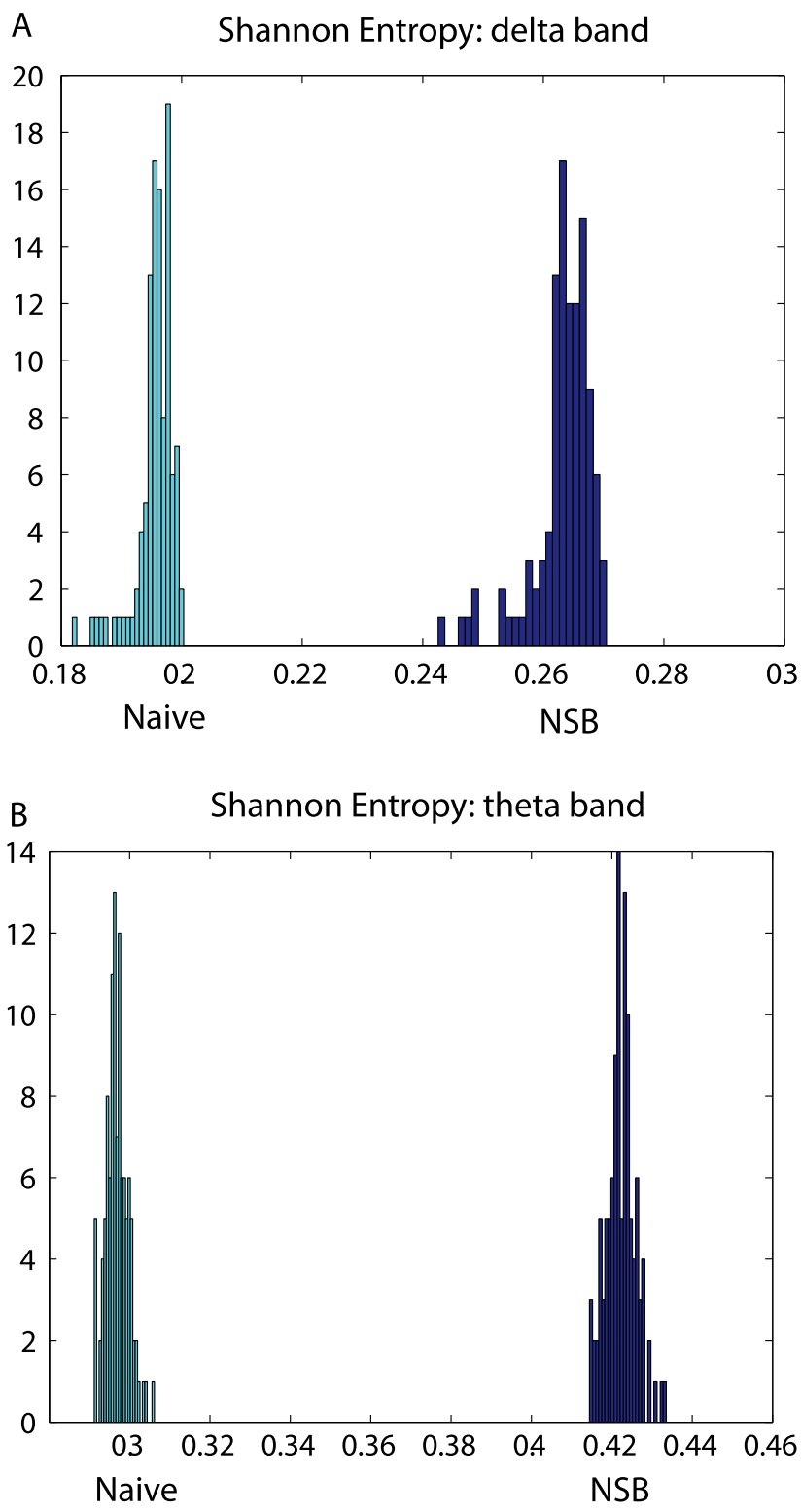

FIG. 14. Shannon entropy histograms considering one of the electrodes. (a) corresponds to the delta band and (b) corresponds to the theta band. We consider $D=6$ and $\tau=1$.

of the normalized Shannon entropy

$$
H[P]=S[P] / S_{\max },
$$

with $S_{\max }=S\left[P_{e}\right]=\log _{2} N,(0 \leq H \leq 1)$, and the disequilibrium $\mathcal{Q}_{J}$ defined in terms of the Jensen-Shannon divergence (JSD). That is,

$$
\mathcal{Q}_{J}\left[P, P_{e}\right]=Q_{0} \mathcal{J}\left[P, P_{e}\right]
$$

with

$$
\mathcal{J}\left[P, P_{e}\right]=S\left[\left(P+P_{e}\right) / 2\right]-S[P] / 2-S\left[P_{e}\right] / 2,
$$

the above-mentioned Jensen-Shannon divergence and $Q_{0}$, a normalization constant $\left(0 \leq \mathcal{Q}_{J} \leq 1\right)$, are equal to the inverse of the maximum possible value of $\mathcal{J}\left[P, P_{e}\right]$. This value is obtained when one of the components of $P$, like $p_{m}$, is equal to one and the remaining $p_{j}$ are equal to zero. The JensenShannon divergence, which quantifies the difference between
A
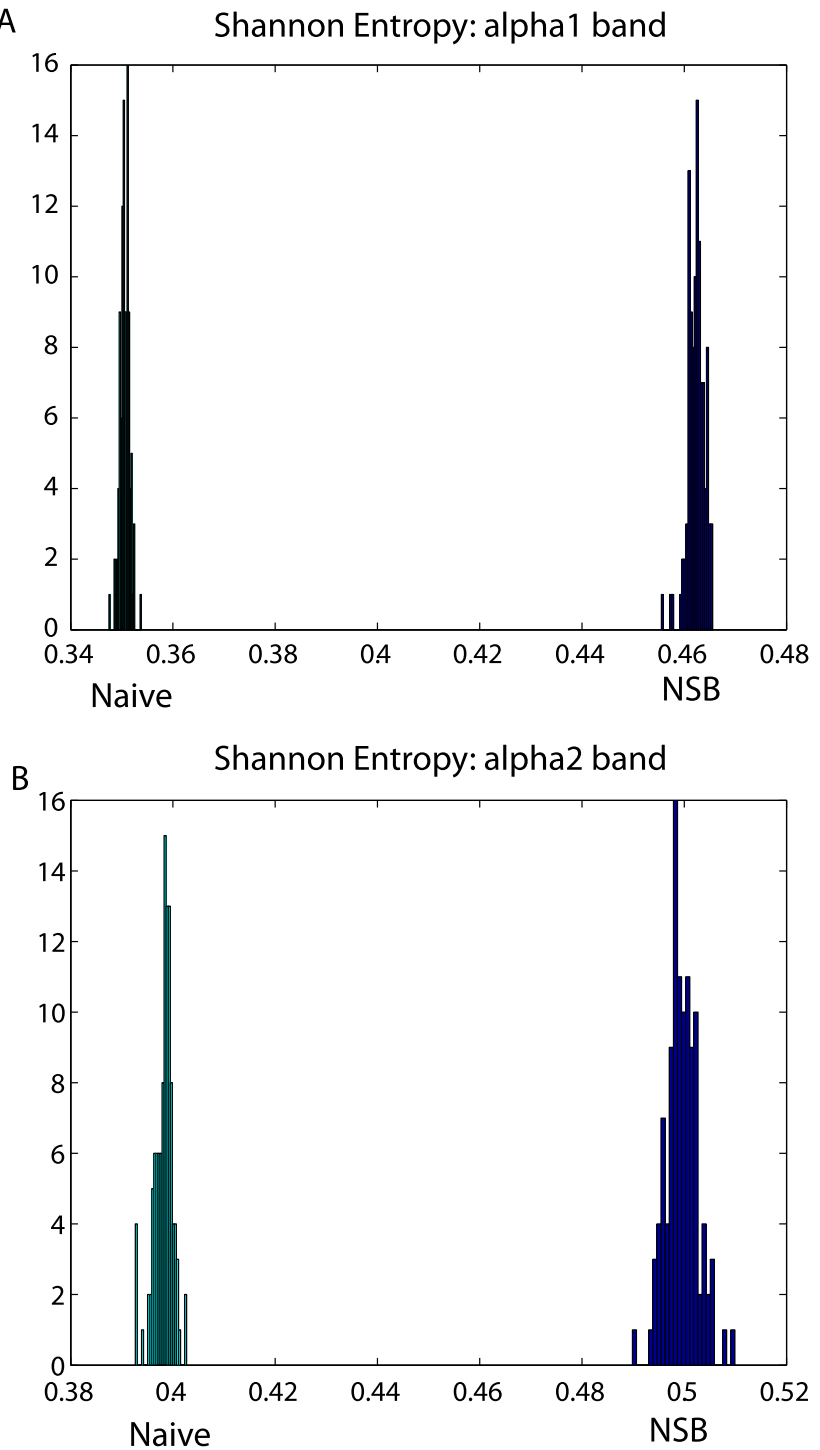

FIG. 15. Shannon entropy histograms considering one of the electrodes. (a) corresponds to the alpha 1 band and (b) corresponds to the alpha 2 band. We consider $D=6$ and $\tau=1$.

probability distributions, is very useful to compare the symbolic composition between different sequences. ${ }^{68}$ The above introduced statistical complexity measure (SCM) depends on two different probability distributions, the one associated with the system under analysis, $P$, and the uniform distribution, $P_{e}$.

Bandt and Pompe (BP) introduced a successful methodology for the evaluation of PDF associated with scalar time data using a symbolization technique. ${ }^{33}$ For a detailed description of the approach, we refer the reader to Ref. 69. The relevant symbolic data are (1) created by ranking the values of the series and (2) defined by reordering the embedded data in ascending order, that is equivalent to a phase space reconstruction with embedding dimension $D$ and time lag $\tau$. Further details describing the advantages that make the Bandt and Pompe methodology more convenient than conventional methods based on range partitioning (i.e., PDF based on histograms) can be found in Refs. 35, 36, 51 and 70-72.

The BP methodology can be applied to any type of time series and the only condition for the applicability 
of this methodology is a very weak stationary assumption (that is, for $k \leq D$, the probability for $x_{t}<x_{t+k}$ should not depend on $t) .{ }^{33}$ To use the Bandt and Pompe ${ }^{33}$ methodology for evaluating the PDF, $P$, associated with the time series, one begins considering partitions of the pertinent $D$-dimensional space that will "reveal" relevant details of the ordinal structure of a given one-dimensional time series $\mathcal{X}(t)=\left\{x_{t} ; t=1, \ldots, M\right\}$ with embedding dimension $D>1$ $(D \in \mathbb{N})$ and embedding time delay $\tau(\tau \in \mathbb{N})$. Let us consider the "ordinal patterns" of order (length) $D$ generated by $(s) \mapsto\left(x_{s-(D-1) \tau}, x_{s-(D-2) \tau}, \ldots, x_{s-\tau}, x_{s}\right)$, that confers to each time $s$ the $D$-dimensional vector of values at times $s, s-$ $\tau, \ldots, s-(D-1) \tau$. Notice that when the $D$-value is bigger more information about the past is included into our vectors. We denote "ordinal pattern" related to the time $(s)$ to the permutation $\pi=\left(r_{0}, r_{1}, \ldots, r_{D-1}\right)$ of $[0,1, \ldots, D-1]$ defined by $x_{s-r_{D-1} \tau} \leq x_{s-r_{D-2} \tau} \leq \cdots \leq x_{s-r_{1} \tau} \leq x_{s-r_{0} \tau}$. Importantly, to get a unique result we take $r_{i}<r_{i-1}$ if $x_{s-r_{i}}=x_{s-r_{i-1}}$. This can be justified if the values of $x_{t}$ have a continuous distribution, so the same values are very unusual. Therefore, for all permutations $D$ possible $\pi$ of order $D$ their natural relative frequencies can of course be calculated how often this particular order in the time series is divided by the total number of episodes.

$$
p\left(\pi_{i}\right)=\frac{\sharp\left[s \mid s \leq N-(D-1) \tau ;(s) \text { has type } \pi_{i}\right]}{N-(D-1) \tau} .
$$

We refer the symbol $\sharp$ to "number". That is, given an ordinal pattern, probability distribution $P=\left[p\left(\pi_{i}\right), i=1, \ldots, D !\right]$ is obtained from the time series. Thus, it is possible to quantify the diversity of the patterns of length $D$ derived from a scalar time series, by evaluating the so-called permutation Shannon entropy and permutation MPR statistical complexity. The embedding dimension $D$ determines the number of accessible states $D$ ! The conditions the minimum acceptable length to work with reliable statistics are $M \gg D$ !, of the time series that one needs in order. ${ }^{51}$ Let us emphasize that Bandt and Pompe suggested working with $4 \leq D \leq 6$ and specifically considered an embedding delay $\tau=1$ in their cornerstone paper. ${ }^{33}$ However, other values of $\tau$ can also provide additional information. . $, 37,70,73-76$

\section{NSB estimations: Removing sample size dependent bias}

The problem of measurements of entropy is that they depend indeed on a limited number of samples provided by a given experiment. Therefore, we need to use a theoretical approach that can remove sample size dependent bias from the entropy estimations. However, approaches that are commonly used to estimate the entropy tend to underestimate this quantity as they are biased.

Long time ago, Ma proposed the idea of calculating the entropy for physical systems by counting coincidences in the micro-canonical ensemble, ${ }^{77}$ where a uniform distribution of entropy corresponds to states of fixed energy. The Bayesian prior proposed by Nemenman et al. ${ }^{32}$ extends this idea of counting coincidences to an arbitrarily complex distribution.
More specifically, the goal of the NSB method is to construct a Bayesian prior, which generates a nearly uniform distribution of entropies in order to correct sample size dependent bias at its source. ${ }^{31,32,78}$ The Bayesian approach ${ }^{31}$ is also based on the formalism formulated by Wolpert and Wolf ${ }^{79}$ and Samengo ${ }^{80}$ extending the entropy estimations counting coincidences to arbitrarily complex distributions. ${ }^{32}$

In the following, we review the basic ideas of the NSB entropy estimator method presented by Nemenman et al. ${ }^{32}$ Let us consider the problem of estimating the Shannon entropy for a given probability distribution $\mathbf{p}=\left\{p_{i}\right\}$.

$$
H=-\sum_{i=1}^{K} p_{i} \log p_{i}
$$

where the index $i$ runs over $K$ possibilities. Consider $N$ samples (trials) which were obtained from a given experiment, where each possibility $i$ occurred $n_{i}$ times: if $N$ is much bigger than $K$, we can approximate

$$
p_{i} \approx f_{i}=n_{i} / N
$$

and therefore, the entropy can be expressed in terms of the observed frequencies as

$$
H_{\text {naive }}=-\sum_{i=1}^{K} f_{i} \log _{2} f_{i}
$$

This "plugin" approach tends to underestimate the entropy. Several attempts to solve this problem were made by Carlton, ${ }^{81}$ who made asymptotic bias corrections by adding a term of order $O(K / N)$. This approach was developed further by Panzeri et al., ${ }^{82}$ and amounts to

$$
H=H_{\text {naive }}+\operatorname{Bias}[H(R)],
$$

where

$$
\operatorname{Bias}[H(R)] \approx-\frac{1}{2 N \log _{2}(2)} \sum_{s} \hat{R}
$$

and $\hat{R}$ denotes the number of relevant bins for the probability distribution $P$, i.e., the number of bins with non-zero probability of occupancy. Panzeri and Treves ${ }^{82}$ made use of a Bayesian prior for the number of relevant bins, and iteratively re-estimated $i^{83,84}$, however, the election of the bins is a very intricate issue and is strongly dependent upon data characteristics.

Different approaches for estimating entropies without using such an asymptotic expansion approach have recently been presented. ${ }^{32,85}$ As stated above, we follow the approach of Nemenman et al. ${ }^{32}$

Let us recall the examination of the probability distribution $\mathbf{p}$ from Eq. (A9). Bayes' rule tells us that we can express the posterior probability of $\mathbf{p}\left[=\left(p_{i}\right)\right]$, given that we have just observed $i$ to have occurred $n_{i}$ times,

$$
P\left(p \mid n_{i}\right)=\frac{P\left(n_{i} \| p\right) P(p)}{P\left(n_{i}\right)} .
$$

Note that the number of times we observe each response value $i$ to occur must add up to the total number of experimental 
trials $N$.

$$
\sum_{i=1}^{K} n_{i}=N
$$

In Eq. (A13), the "prior" distribution is $P(\mathbf{p})$ - in principle, we could choose a prior such that our estimator of the entropy of $P(\mathbf{p})$ does not depend upon the number of trials. In order to achieve this goal, we can express $P$ (p) in terms of the Dirichlet family of priors. ${ }^{32}$ This should allow us to construct a prior which does not depend on inverse powers of $N$ (which even the naive approximation does implicitly, as $\left.f_{i}=n_{i} / N\right)$. The Dirichlet family are characterized by a parameter $\beta$; they can be written as

$$
P_{\beta}(p)=\frac{1}{Z} \delta\left(1-\sum_{i=1}^{K} p_{i}\right) \prod_{i=1}^{K} p_{i}^{\beta-1},
$$

where

$$
Z=\frac{\Gamma^{K}(\beta)}{\Gamma(K \beta)},
$$

and $\delta$ and $Z$ are functions which enforce the normalization of $\mathbf{p}$ and $P_{\beta}$, respectively ( $\delta$ being the Dirac delta function). Maximum likelihood estimation corresponds to Bayesian estimation with this prior in the limit $\beta \rightarrow 0$, while a uniform prior is implemented by $\beta=1$. Nemenman et al..$^{31}$ observed that fixing a particular value of $\beta$ (and thus fixing the prior) specifies the entropy almost exactly. For an "incorrect" prior, the estimate of entropy is thus dominated by the prior, rather than determined by our actual knowledge - that is, it is biased.

Ideally, we would like to compute the whole a priori distribution of entropies

$$
P_{\beta}(H)=\int d p_{1} d p_{2} \cdots d p_{K} P_{\beta}\left[\left(p_{i}\right)\right] \delta\left(H+\sum_{i=1}^{K} p_{i} \log _{2} p_{i}\right) .
$$

But this is very difficult to achieve. In order to get an entropy estimate with small bias, one could simply fix a flat prior distribution of entropy $P(H)$. One way of doing so is by defining (see Ref. 32)

$$
P(H)=1=\int \delta(H-\xi) d \xi,
$$

where $\xi$ is the expected entropy. If we could find a family of priors $P_{\beta}(\mathbf{p})$ which result in $\delta$ functions over $H$, and if by changing $\beta$ we move the peak across the whole range of entropies uniformly, then we will effectively be choosing the proper prior for each entropy. We will thus come close to the objective of removing bias at its source. Because the entropy of distributions chosen for $P_{\beta}$ is sharply defined and monotonically dependent on the parameter $\beta$, we can effect this goal by averaging over $\beta$. The main idea of the NSB approach is thus to construct a prior

$$
P_{\beta}(p)=\frac{1}{Z} \delta\left(1-\sum_{i=1}^{K} p_{i}\right) \prod_{i=1}^{K} p_{i}^{\beta-1} \frac{d \xi(\beta)}{d \beta} P(\beta),
$$

which will perform this task even when $N$ is small. In this equation, $\mathrm{Z}$ is again a normalizing coefficient and $\frac{d \xi(\beta)}{d \beta}$ ensures the uniformity for a priori expected entropy $\xi$.
This Dirichlet priors allow all the $K$ dimensional integrals to be calculated analytically. ${ }^{32,79}$ Thus, the moments of the entropy reads as

$$
\left(H^{N S B}\right)^{m}=\frac{\int d \xi \rho(\xi, n)\left\langle H^{m}(n)\right\rangle_{\beta(\xi)}}{\int d \xi \rho(\xi, n)},
$$

where $\mathbf{n}=\left\{n_{i}\right\}$, and $m=1,2$ correspond to the entropy and its second moment. $\left\langle H^{m}\left[n_{i}\right]\right\rangle_{\beta(\xi)}$ is the expectation value of the $m$ th entropy moment at a fixed $\beta^{79}$ and the posterior density is a function of the proposed Dirichlet prior

$$
\rho(\xi \mid n)=P_{\beta}(\xi) \frac{\Gamma[\kappa(\xi)]}{\Gamma[\beta(\xi)]} \prod_{i=1}^{K} \frac{\Gamma\left[n_{i}+\beta(\xi)\right]}{\Gamma[\beta(\xi)]} .
$$

For further details of the NSB methodology, see Refs. 31 and 32.

Let us now recall that the Jensen-Shannon divergence (JSD) is defined as ${ }^{86}$

$$
J S(\mathrm{P}, \mathrm{Q})=\frac{1}{2}\left[D_{K L}\left(\mathrm{P} \mid \frac{\mathrm{P}+\mathrm{Q}}{2}\right)+D_{K L}\left(\mathrm{Q} \mid \frac{\mathrm{P}+\mathrm{Q}}{2}\right)\right],
$$

where $D_{K L}$ is the Kullback-Leibler divergence between the two distributions, and thus Eq. (A22) can be rewritten in terms of entropy-like quantities. We take $\mathrm{P} \equiv P_{\beta}$ as the full probability distribution and $\mathrm{Q} \equiv P_{e}$ as the independent distribution. It is therefore straightforward, from our previous description, how to apply the NSB methodology to remove sample size bias from the entropy, Eq. (A4), and complexity estimations, Eq. (A3).

Thus, we can combine the BP methodology $33,35,36$ with the NSB algorithm ${ }^{31,32}$ to estimate the statistical complexity, Eq. (A3), and the entropy, Eq. (A4), avoiding bias deviations due to the finite size of the data. Figures 14(a), 14(b), 15(a), and 15(b) show the histograms of the Shannon entropy for one of the electrodes with and without the NSB methodology considering the different oscillation bands: delta, theta, alpha 1, and alpha 2, respectively. Figures 16(a), 16(b), 17(a), and 17(b) show the histograms of the Shannon entropy for one of the electrodes with and without the NSB methodology considering the different oscillation bands: beta 1 , beta 2 , gamma 1, and gamma 2, respectively. Figures 18(a), 18(b), 19(a), and 19(b) show the histograms of the MPR complexity for one of the electrodes with and without the NSB methodology considering the different oscillation bands: delta, theta, alpha 1, and alpha 2, respectively. Figures 20(a), 20(b), 21(a), and 21(b) show the histogram of the MPR complexity for one of the electrodes with and without the NSB methodology considering the different oscillation bands: beta 1, beta 2 , gamma 1, and gamma 2, respectively. Moreover, the main idea of NSB is to construct a Bayesian prior, which generates a nearly uniform distribution of entropies in order to avoid bias at its origin. These results were obtained by Nemenman et al. $; 31,32$ we provided in this section a simple explanation of how to combine the BP methodology $y^{33,35,36}$ with the NSB algorithm ${ }^{31,32}$ to estimate the information quantifiers removing bias deviations. We applied the NSB method ${ }^{31,32}$ to remove bias deviations of the entropy and complexity measures when using EEGs data, and that are estimated using the 

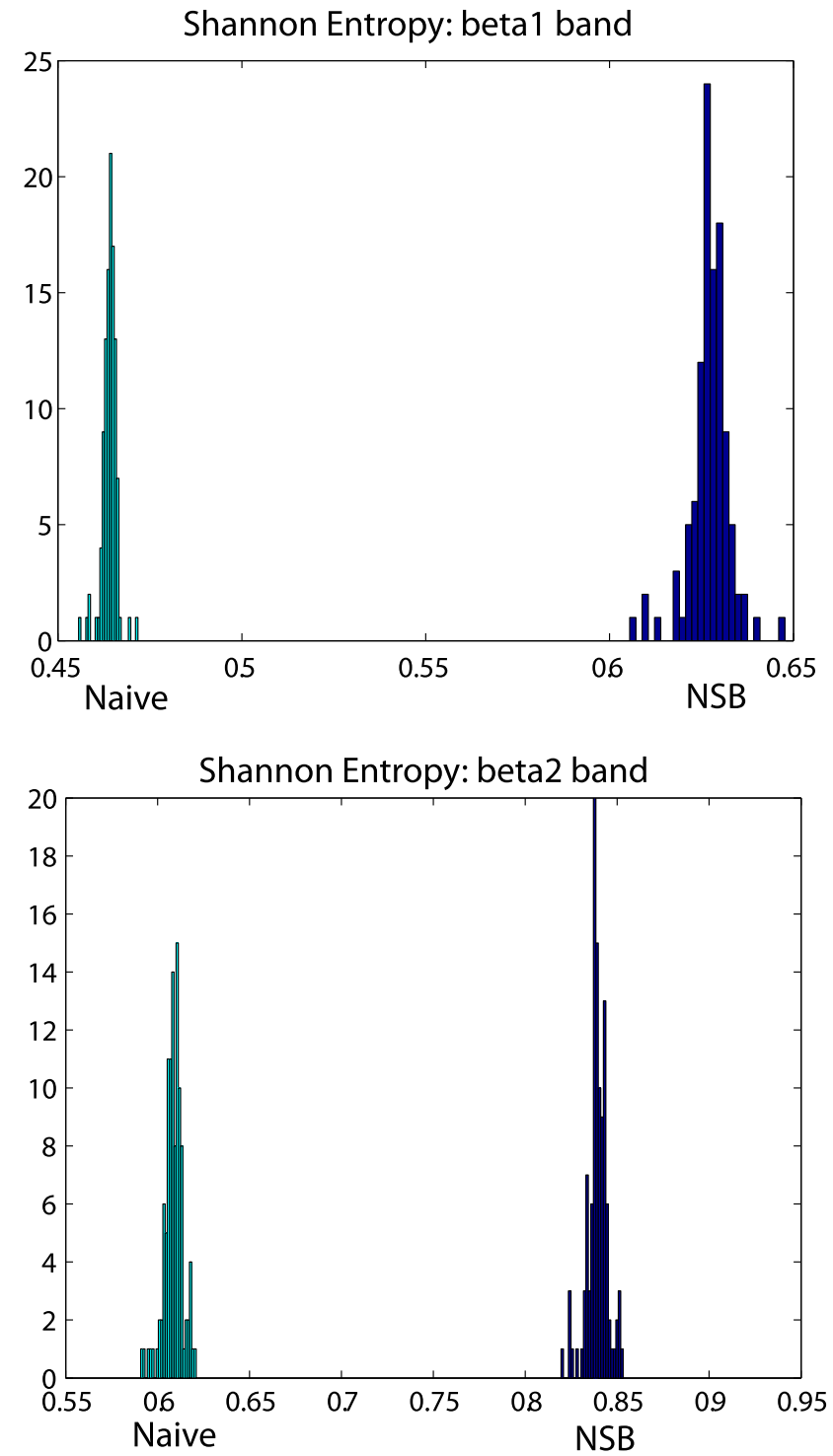

FIG. 16. Shannon entropy histograms considering one of the electrodes. (a) corresponds to the beta 1 band and (b) corresponds to the beta 2 band. We consider $D=6$ and $\tau=1$.

BP approach. ${ }^{33,35,36}$ Details about the performance of the NSB method can be found in Refs. 27, 31, and 32.

\section{Experimental protocol}

We have considered the EEG Motor Movement/Imagery Dataset recorded using $\mathrm{BCI} 2000^{21}$ instrumentation system available through Physionet. ${ }^{34,40-42}$ The dataset consists of more than 1500 EEG recordings, with different durations (one or two minutes per record), obtained from 109 healthy subjects. The data of the 109 subjects on Physionet are screening data in which the subjects had to perform one movement or imagery task at a time. The population of volunteers was drawn from the employer at the New York State Department of Health, i.e., all the subjects had completed high school training and most of them had completed four years of college education. The sex and age of the subjects have not been reported as relevant for performing these tasks. All subjects were naive. As learning proceeded with further repetitions of
A Shannon Entropy: gamma1 band

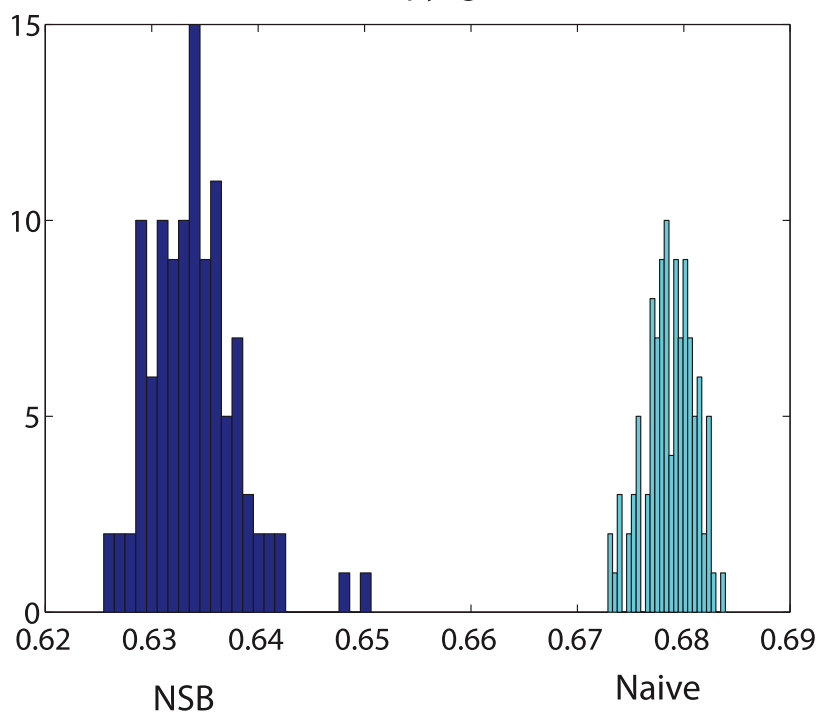

B Shannon Entropy: gamma2 band

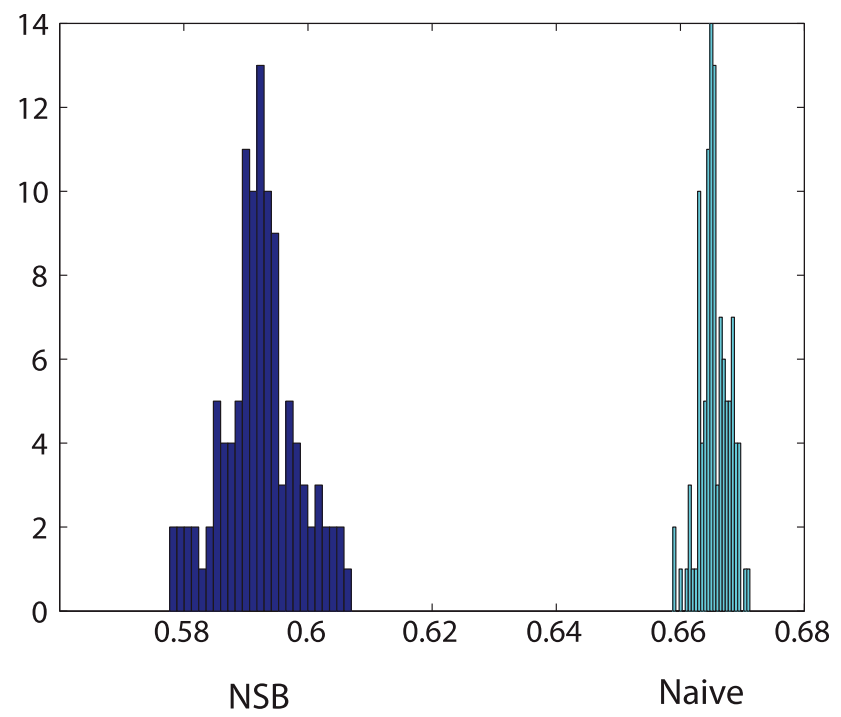

FIG. 17. Shannon entropy histograms considering one of the electrodes. (a) corresponds to the gamma 1 band and (b) corresponds to the gamma 2 band. We consider $D=6$ and $\tau=1$.

the experiment, imagery usually became less important and performance became more automatic. ${ }^{34,40-44}$ Subjects were asked to perform different motor/imagery tasks while EEG signals were recorded from 64 electrodes along the surface of the scalp. Each subject performed 14 experimental runs:

- A 1-min baseline runs (with eyes open)

- A 1-min baseline runs (with eyes closed)

- Three 2-min runs of each of the four following tasks:

1. A target appears on either the left or the right side of the screen. The subject opens and closes the corresponding fist until the target disappears. Then the subject relaxes.

2. A target appears on either the left or the right side of the screen. The subject imagines opening and closing the corresponding fist until the target disappears. Then the subject relaxes. 
A Complexity: delta band
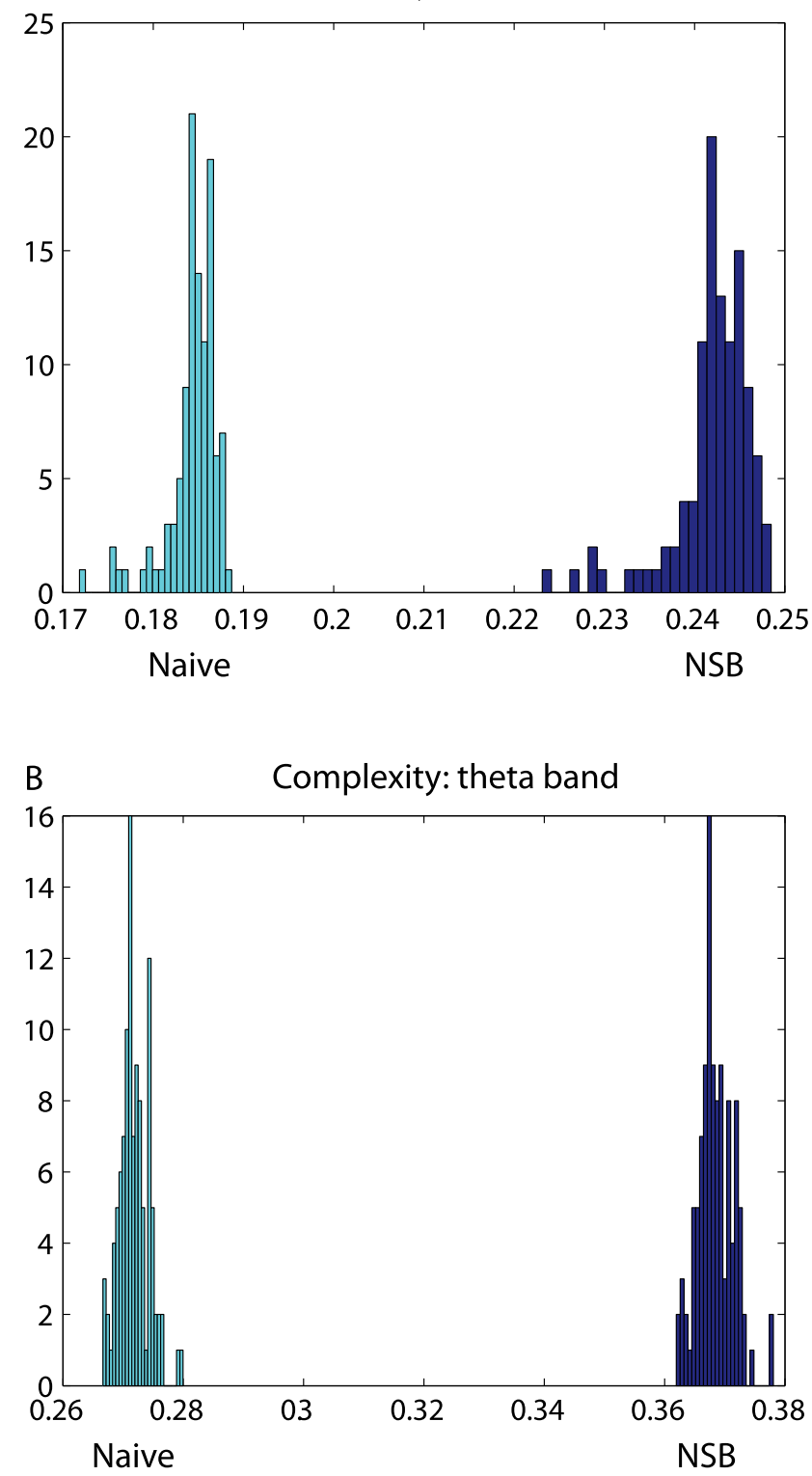

FIG. 18. Complexity histograms considering one of the electrodes. (a) corresponds to the delta band and (b) corresponds to the theta band. We consider $D=6$ and $\tau=1$.

3. A target appears on either the top or the bottom of the screen. The subject opens and closes either both fists (if the target is on the top) or both feet (if the target is on the bottom) until the target disappears. Then the subject relaxes.

4. A target appears on either the top or the bottom of the screen. The subject imagines opening and closing either both fists (if the target is on the top) or both feet (if the target is on the bottom) until the target disappears. Then the subject relaxes.

The 64 channel EEG signals were recorded according to the international 10-20 system (as seen in Fig. 1. The sampling frequency of the EEG is $160 \mathrm{~Hz}$. The order of the recordings are baseline with open eyes, baseline with closed eyes, and then task 1 , task 2 , task 3 , and task 4 successively until
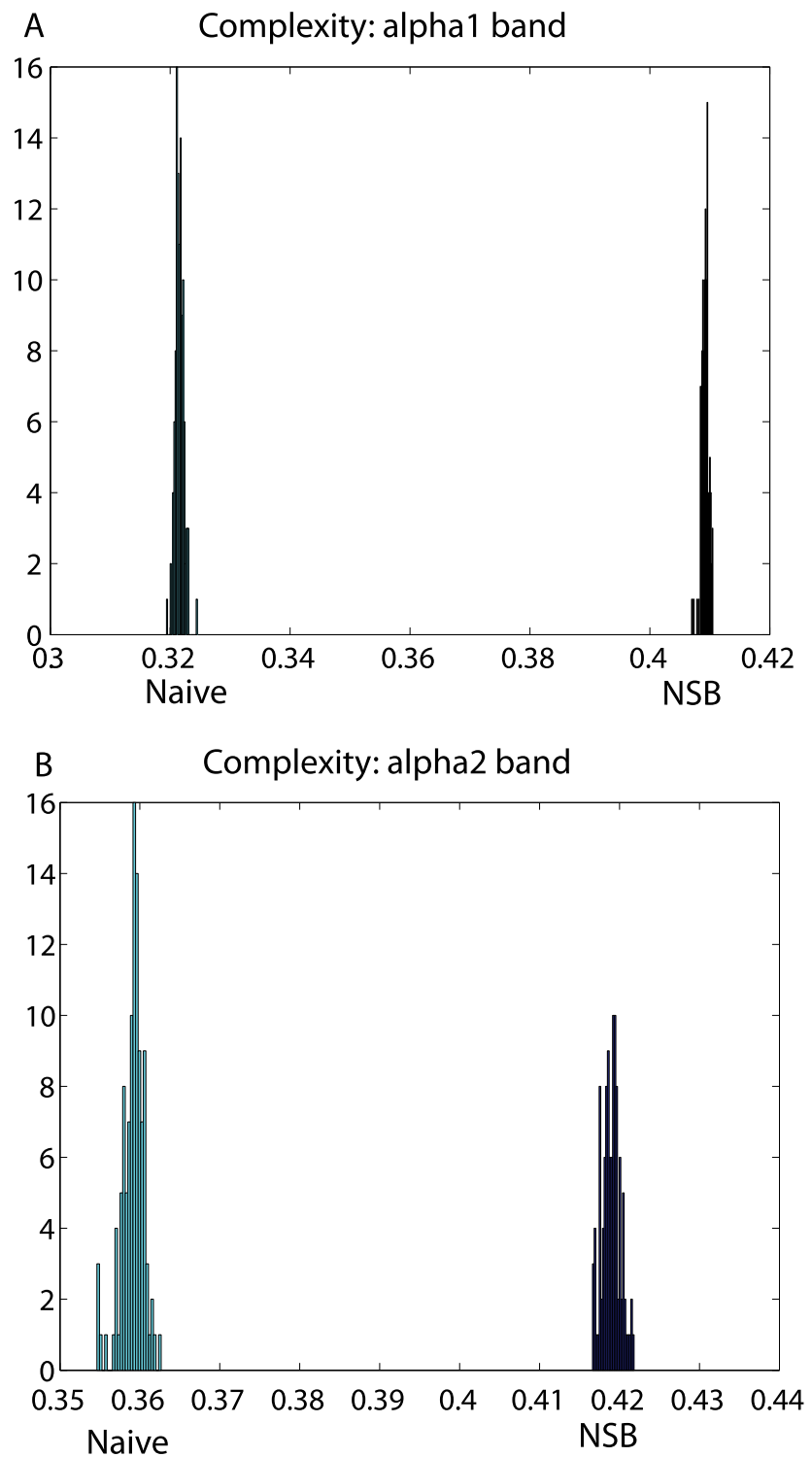

FIG. 19. Complexity histograms considering one of the electrodes. (a) corresponds to the alpha 1 band and (b) corresponds to the alpha 2 band. We consider $D=6$ and $\tau=1$.

the 14 runs where completed. The right mastoid bone corresponds to ground (GND) and the right earlobe to reference (REF). ${ }^{21,34,41-44}$ The impedance was kept under $10 \mathrm{kOhm}$. The data are raw data without any re-referencing. There was no electrooculogram (EOG) recording. The data are raw without any post-processing. A variety of sensors for monitoring brain activity were used. Muscular artifacts [electromyographic (EMG) signals] were carefully checked at the beginning of each recording and verified throughout the recording. During the recordings, light was dimmed, and the experiment was performed in a closed room, so it minimized external sounds. As the learning proceeds, imagery usually becomes less important. ${ }^{21,34,40-44}$ The timing from the event markers was of $1 \mathrm{~s}$ and they are stated in the dataset (see Ref. 42, the tasks order was block-randomized, in blocks of 8 . For further details of the EEG data acquisition, we refer the reader to Refs. 21, 34, and 41-44. 


\section{A}

Complexity: beta1 band

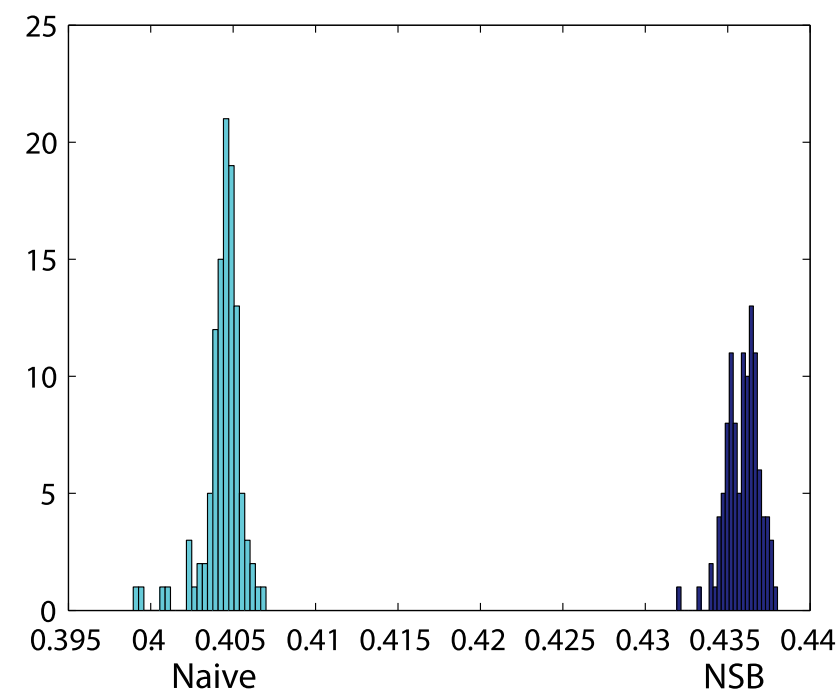

B Complexity: beta2 band

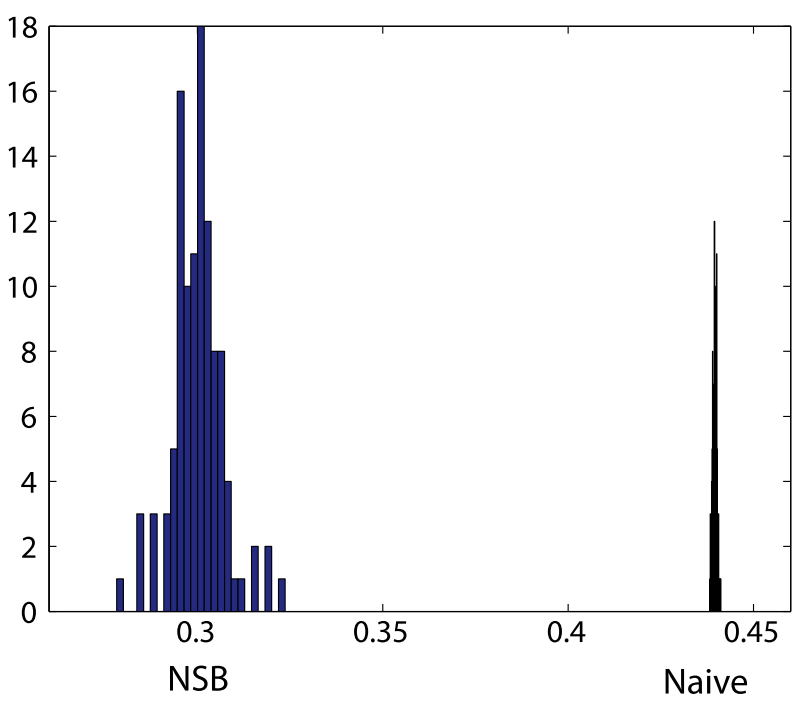

FIG. 20. Complexity histograms considering one of the electrodes. (a) corresponds to the beta 1 band and (b) corresponds to the beta 2 band. We consider $D=6$ and $\tau=1$.

\section{Data recording and processing}

The EEGs were recorded from 64 electrodes as per the international 10-20 system, as shown in Fig. 1(a) (see experimental setup of the BCI2000 system ${ }^{21,34}$ ).

The sampling frequency of the EEG is $160 \mathrm{~Hz}$. But because of the high frequency artifacts that blurred the EEG, and to remove fluctuations at DC level and increase the signal resolution, the records were filtered first between 1 and $50 \mathrm{~Hz}$ using a filter based on the Kaiser window developed by Belitski et al. ${ }^{45}$ with sharp transition bandwidth $(0.1 \mathrm{~Hz})$, small passband ripple $(0.05 \mathrm{~dB})$, and high stopband attenuation $(60 \mathrm{~dB})$.

After this filtering, a bandpass filtering were made. The bands in consideration were given in Table I.

Each signal has 20000 points, thus we use the BP method in combination with the NSB algorithm that

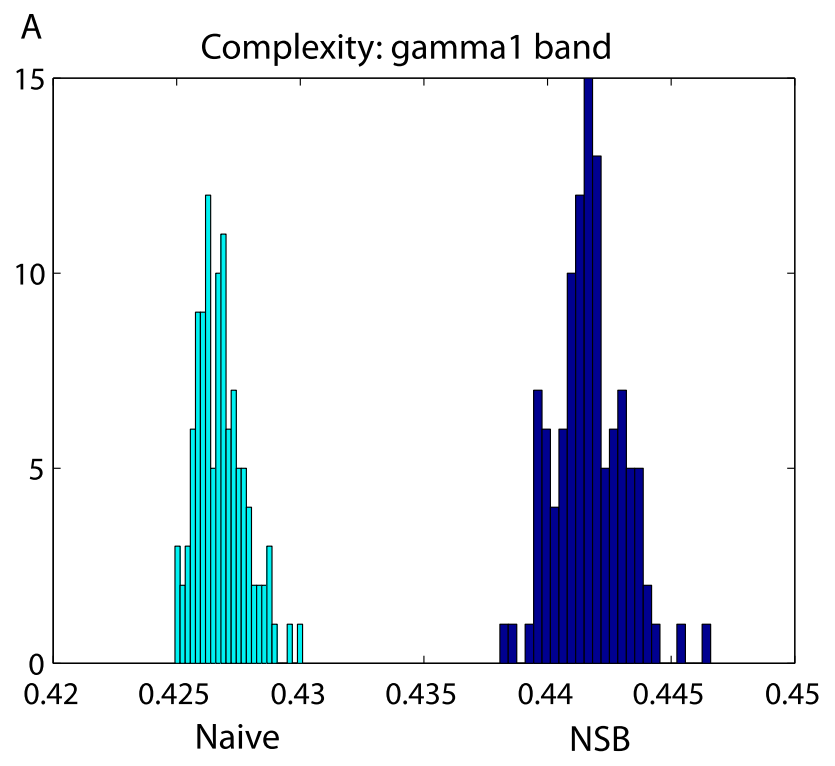

B

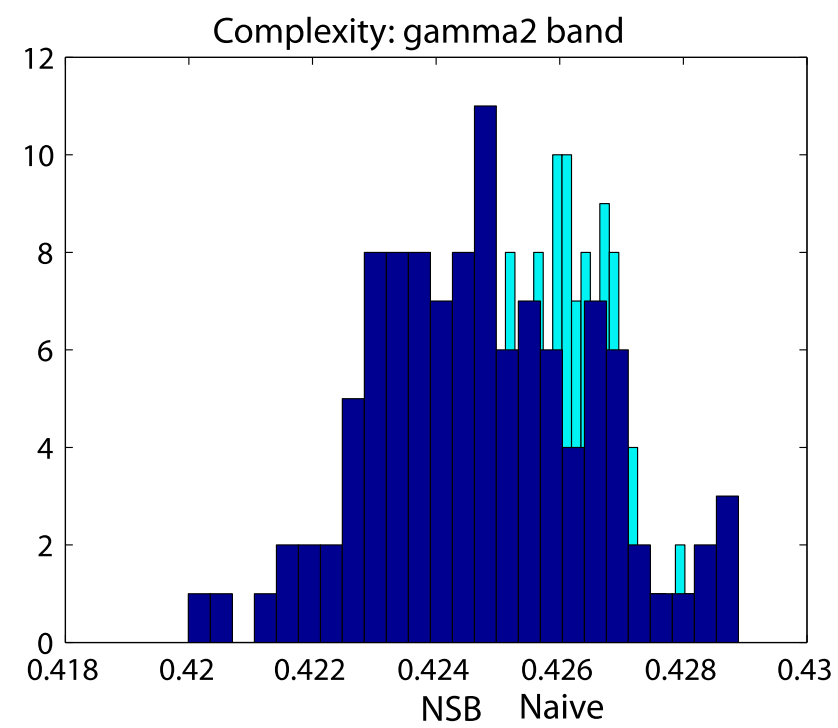

FIG. 21. Complexity histograms considering one of the electrodes. (a) corresponds to the gamma 1 band and (b) corresponds to the gamma 2 band. We consider $D=6$ and $\tau=1$.

TABLE I. Frequency bands analysed.

\begin{tabular}{lc}
\hline \hline Band & Frequency interval $(\mathrm{Hz})$ \\
\hline Delta & {$[1,4)$} \\
Theta & {$[4,8)$} \\
Alpha 1 & {$[8,10)$} \\
Alpha 2 & {$[10,13)$} \\
Beta 1 & {$[13,18)$} \\
Beta 2 & {$[18,31)$} \\
Gamma 1 & {$[31,41)$} \\
Gamma 2 & {$[41,50)$} \\
\hline \hline
\end{tabular}

guarantees estimations of entropy and complexity free of bias deviations.

${ }^{1}$ G. Buzsáki, Rhythms of the Brain, (Oxford University Press, New York, USA, 2006).

${ }^{2}$ F. Montani and O. Rosso, "Entropy-complexity characterization of brain development in chickens," Entropy 16, 4677-4692 (2014). 
${ }^{3}$ A. Kostov and M. Polak, "Parallel man-machine training in development of EEG-based cursor control," IEEE Trans. Rehabil. Eng. 8, 203-205 (2000).

${ }^{4}$ D. McFarland, G. W. Neat, R. F. Read, and J. R. Wolpaw, "An EEG-based method for graded cursor control," Psychobiology 21, 77-81 (1993).

${ }^{5}$ J. d. R. Millán, F. Renkens, J. Mourino, and W. Gerstner, "Noninvasive brain-actuated control of a mobile robot by human EEG," IEEE Trans. Biomed. Eng. 51, 1026-1033 (2004).

${ }^{6} \mathrm{G}$. Pfurtscheller, D. Flotzinger, and J. Kalcher, "Brain-computer interface: A new communication device for handicapped persons," J. Microcomput. Appl. 16, 293-299 (1993).

${ }^{7}$ J. Wolpaw, D. McFarland, G. Neat, and C. Forneris, "An EEG-based brain-computer interface for cursor control," Electroencephalogr. Clin. Neurophysiol. 78, 252-259 (1991).

${ }^{8}$ J. Wolpaw and D. McFarland, "Multichannel EEG-based brain-computer communication," Electroencephalogr. Clin. Neurophysiol. 90, 444-449 (1994).

${ }^{9}$ J. Wolpaw and D. McFarland, "Control of a two-dimensional movement signal by a noninvasive brain-computer interface in humans," Proc. Natl. Acad. Sci. U.S.A. 101, 17849-17854 (2004).

${ }^{10}$ E. Felton, J. Wilson, J. Williams, and P. Garell, "Electrocorticographically controlled brain-computer interfaces using motor and sensory imagery in patients with temporary subdural electrode implants. Report of four cases," J. Neurosurg. 106, 495-500 (2007).

${ }^{11}$ E. Leuthardt, G. Schalk, J. Ojemann, and D. Moran, "A brain-computer interface using electrocorticographic signals in humans," J. Neural Eng. 1, 63-71 (2004).

${ }^{12}$ E. Leuthardt, K. Miller, G. Schalk, R. Rao, and J. Ojemann, "Electrocorticography-based brain computer interface: The seattle experience,” IEEE Trans. Neural Syst. Rehabil. Eng. 14(2), 194-198 (2006).

${ }^{13}$ J. Wilson, E. Felton, P. Garell, G. Schalk, and J. Williams, "ECoG factors underlying multimodal control of a brain-computer interface," IEEE Trans. Neural Syst. Rehabil. Eng. 14(2), 246-250 (2006).

${ }^{14}$ N. Crone, D. Miglioretti, B. Gordon, and R. Lesser, "Functional mapping of human sensorimotor cortex with electrocorticographic spectral analysis. ii. Event-related synchronization in the gamma band," Brain 121, 2301-2315 (1998).

${ }^{15}$ J. Lachaux, E. Rodriguez, J. Martinerie, C. Adam, D. Hasboun, and F. Varela, "A quantitative study of gamma-band activity in human intracranial recordings triggered by visual stimuli,” Eur. J. Neurosci. 12, 2608-2622 (2000).

${ }^{16}$ J. Lachaux, P. Fonlupt, P. Kahane, L. Minotti, D. Hoffmann, O. Bertrand, and M. Baciu, "Relationship between task-related gamma oscillations and bold signal: New insights from combined fMRI and intracranial EEG," Hum. Brain Mapp. 28, 1368-1375 (2007).

${ }^{17}$ K. Miller, E. Leuthardt, G. Schalk, R. Rao, N. Anderson, D. Moran, J. Miller, and J. Ojemann, "Spectral changes in cortical surface potentials during motor movement," J. Neurosci. 27, 2424-2432 (2007).

${ }^{18}$ T. Pistohl, T. Ball, A. Schulze-Bonhage, A. Aertsen, and C. Mehring, "Prediction of arm movement trajectories from ECoG-recordings in humans," J. Neurosci. Methods 167, 105-114 (2008).

${ }^{19}$ J. Sanchez, A. Gunduz, P. Carney, and J. Principe, "Extraction and localization of mesoscopicmotor control signals for human ECoG neuroprosthetics," J. Neurosci. Methods 167, 63-81 (2008).

${ }^{20}$ G. Schalk, J. Kubánek, K. Miller, N. Anderson, E. Leuthardt, J. Ojemann, D. Limbrick, D. Moran, L. Gerhardt, and J. Wolpaw, "Decoding twodimensional movement trajectories using electrocorticographic signals in humans," J. Neural Eng. 4, 264-275 (2007).

${ }^{21}$ G. Schalk and J. Mellinger, A Practical Guide to Brain-Computer Interfacing with BCI2000 (Springer London Dordrecht Heidelberg New York, 2010).

${ }^{22}$ J. Lachaux, D. Hoffmann, L. Minotti, A. Berthoz, and P. Kahane, "Intracerebral dynamics of saccade generation in the human frontal eye field and supplementary eye field," Neuroimage 30, 1302-1312 (2006).

${ }^{23}$ F. Aoki, F. Fetz, L. Shupe, E. Lettich, and G. Ojemann, "Changes in power and coherence of brain activity in human sensorimotor cortex during performance of visuomotor tasks," Biosystems 63, 89-99 (2001).

${ }^{24}$ J. Fell, P. Klaver, K. Lehnertz, T. Grunwald, C. Schaller, C. Elger, and G. Fernandez, "Human memory formation is accompanied by rhinalhippocampal coupling and decoupling," Nat. Neurosci. 4, 1259-1264 (2001).
${ }^{25}$ G. Chen, M. Rasch, R. Wang, and X. Zhang, "Experience-dependent emergence of beta and gamma band oscillations in the primary visual cortex during the critical period," Sci. Rep. 5, 17847 (2015).

${ }^{26}$ K. Tanji, K. Suzuki, A. Delorme, H. Shamoto, and N. Nakasato, "High-frequency gamma-band activity in the basal temporal cortex during picture-naming and lexical-decision tasks," J. Neurosci. 25, 3287-3293 (2005).

${ }^{27}$ F. Montani, A. Kohn, M. Smith, and S. Schultz, "The role of correlations in direction and contrast coding in the primary visual cortex," J. Neurosci. 27, 2338-2348 (2007).

${ }^{28}$ F. Montani, R. Ince, R. Senatore, E. Arabzadeh, M. Diamond, and S. Panzeri, "The impact of high-order interactions on the rate of synchronous discharge and information transmission in somatosensory cortex," Philos. Trans. R. Soc. A 367, 3297-310 (2009).

${ }^{29}$ S. Panzeri, R. Senatore, M. A. Montemurro, and R. Petersen, "Correcting for the sampling bias problem in spike train information measures," J. Neurophys. 98, 1064-1072 (2007).

${ }^{30}$ F. Montani, A. Oliynyk, and L. Fadiga, "Superlinear summation of information in premotor neuron pairs," Int. J. Neural Syst. 27, 1650009 (2017).

${ }^{31}$ I. Nemenman, F. Shafee, and W. Bialek, Entropy and Inference, Revisited. Adv. Neural Inf. Proc. Syst. Vol. 14, edited by T. G. Dietterich, S. Becker, and Z. Ghahramani (MIT Press, Cambridge, MA, 2002).

${ }^{32}$ I. Nemenman, W. Bialek, and R. Steveninck, "Entropy and information in neuronal spike trains: Progress on the sampling problem,” Phys. Rev. E 69, 056111-056116 (2004).

${ }^{33} \mathrm{C}$. Bandt and B. Pompe, "Permutation entropy: A natural complexity measure for time series." Phys. Rev. Lett. 88, 174102 (2002).

${ }^{34}$ G. Schalk, D. McFarland, T. Hinterberger, N. Birbaumer, and J. Wolpaw, "BCI2000: A general-purpose brain-computer interface (BCI) system," IEEE Trans. Biomed. Eng. 51, 1034-1043 (2004).

${ }^{35} \mathrm{O}$. Rosso and C. Masoller, "Detecting and quantifying stochastic and coherence resonances via information-theory complexity measurements," Phys. Rev. E 79, 040106(R) (2009).

${ }^{36} \mathrm{O}$. Rosso and C. Masoller, "Detecting and quantifying temporal correlations in stochastic resonance via information theory measures," Eur. Phys. J. B 69, 37-43 (2009).

${ }^{37}$ O. Rosso, F. Olivares, and A. Plastino, "Noise versus chaos in a causal Fisher-Shannon plane," Pap. Phys. 7, 070006 (2015).

${ }^{38}$ F. Montani, O. Rosso, F. Matias, S. Bressler, and C. Mirasso, "A symbolic information approach to determine anticipated and delayed synchronization in neuronal circuit models," Philos. Trans. R. Soc. Lond. Ser. A 373, 20150110 (2015).

${ }^{39}$ F. Montani, R. Baravalle, L. Montangie, and O. Rosso, "Causal information quantification of prominent dynamical features of biological neurons," Philos. Trans. R. Soc. Lond. Ser. A 373, 20150109 (2015).

${ }^{40}$ J. R. Wolpaw, D. J. McFarland, T. M. Vaughan, and G. Schalk, "The wadsworth center brain-computer interface (BCI) research and development program," IEEE Trans. Neural Syst. Rehabil. Eng. 11, 1-4 (2003).

${ }^{41}$ A. Goldberger, L. Amaral, L. Glass, J. Hausdorff, P. Ivanov, R. Mark, J. Mietus, G. Moody, C.-K. Peng, and H. Stanley, "Physiobank, physiotoolkit, and physionet: Components of a new research resource for complex physiologic signals," Circulation 101, E215-E220 (2000).

${ }^{42}$ See https://www.physionet.org/pn4/eegmmidb/ for EEG Motor Movement/Imagery Dataset.

${ }^{43}$ N. T. M. Huong, H. Q. Linh, and L. Q. Khai, "Classification of left/right hand movement EEG signals using event related potentials and advanced features," in Sixth International Conference on the Development of Biomedical Engineering in Vietnam (BME6), edited by T. Vo Van, T. Nguyen Le, T. Nguyen Duc, IFMBE Proceedings, (Springer, Singapore, 2017) Vol. 63

${ }^{44}$ G. Zebende, F. Oliveira Filho, and J. Leyva Cruz, "Auto-correlation in the motor/imaginary human eeg signals: A vision about the FDFA fluctuations". PLoS One 12, e0183121 (2017).

${ }^{45}$ A. Belitski, A. Gretton, C. Magri, Y. Murayama, M. Montemurro, N. Logothetis, and S. Panzeri, "Low-frequency local field potentials and spikes in primary visual cortex convey independent visual information," J. Neurosci. 28, 5696-5709 (2008).

${ }^{46} \mathrm{X}$. Wang, "Neurophysiological and computational principles of cortical rhythms in cognition," Physiol. Rev. 90, 1195-1268 (2010). 
${ }^{47}$ C. Hammond, H. Bergman, and P. Brown, "Pathological synchronization in Parkinson's disease: Networks, models and treatments," Trends Neurosci. 30, 357-364 (2007).

${ }^{48}$ G. Deco and A. Thiele, "Attention: Oscillations and neuropharmacology," Eur. J. Neurosci. 30, 347-354 (2009).

${ }^{49} \mathrm{G}$. Thut, C. Miniussi, and J. Gross, "The functional importance of rhythmic activity in the brain," Curr. Biol. 22, 658-663 (2012).

${ }^{50}$ M. Martín, A. Plastino, and O. Rosso, "Generalized statistical complexity measures: Geometrical and analytical properties," Phys. A 369, 439-462 (2006).

${ }^{51}$ O. Rosso, H. Larrondo, M. Martín, A. Plastino, and M. Fuentes, "Distinguishing noise from chaos," Phys. Rev. Lett. 99, 154102 (2007).

${ }^{52}$ D. Feldman, C. McTague, and J. Crutchfield, "The organization of intrinsic computation: Complexity-entropy diagrams and the diversity of natural information processing." Chaos 18, 043106 (2008).

${ }^{53}$ F. Montani, E. Deleglise, and O. Rosso, "Efficiency characterization of a large neuronal network: A causal information approach," Phys. A 401, 58-70 (2014).

${ }^{54}$ C. Kayser, M. Montemurro, N. Logothetis, and S. Panzeri, "Spike-phase coding boosts and stabilizes information carried by spatial and temporal spike patterns," Neuron 61, 597-608 (2009).

${ }^{55}$ V. Sohal, S. Pangratz-Fuehrer, U. Rudolph, and J. Huguenard, "Intrinsic and synaptic dynamics interact to generate emergent patterns of rhythmic bursting in thalamocortical neurons," J. Neurosci. 26, 4247-4255 (2006).

${ }^{56}$ R. Llinas, "Intrinsic electrical properties of mammalian neurons and CNS function: A historical perspective," Front. Cell. Neurosci. 8, 1-14 (2014).

${ }^{57}$ I. Guler and E. Ubeyli, "Multiclass support vector machines for EEGsignals classification," IEEE Trans. Inf. Technol. Biomed. 11, 117-126 (2007).

${ }^{58}$ E. Başar, Chaos in Brain Function, 2nd ed. (Springer Science \& Business Media, 2012).

${ }^{59}$ M. Dafilis, F. Frascoli, P. Cadusch, and D. Liley, "Chaos and generalised multistability in a mesoscopic model of the electroencephalogram," Phys. D 238, 1056-1060 (2009).

${ }^{60}$ O. Rosso, F. Olivares, L. Zunino, L. De Micco, A. Aquino, A. Plastino, and H. Larrondo, "Characterization of chaotic maps using the permutation Bandt-Pompe probability distribution," Eur. Phys. J. B 86, 116 (2013).

${ }^{61}$ W. Klimesch, "Alpha-band oscillations, attention, and controlled access to stored information,” Trends Cogn. Sci. 16, 606-617 (2012).

${ }^{62} \mathrm{C}$. Tallon-Baudry and O. Bertrand, "Oscillatory gamma activity in humans and its role in object representation," Trends Cogn. Sci. 3, 151-162 (1999).

${ }^{63}$ C. Tallon-Baudry, O. Bertrand, F. Peronnet, and J. Pernier, "Induced gamma-band activity during the delay of a visual short-term memory task in humans," J. Neurosci. 18, 4244-4254 (1998).

${ }^{64} \mathrm{C}$. Shannon and W. Weaver, The Mathematical Theory of Communication (University of Illinois Press, Champaign, IL, 1949).

${ }^{65}$ D. Feldman and J. Crutchfield, "Measures of statistical complexity: Why?" Phys. Lett. A 238, 244-252 (1998).

${ }^{66}$ P. Lamberti, M. Martín, A. Plastino, and O. Rosso, "Intensive entropic nontriviality measure," Phys. A 334, 119-131 (2008).
${ }^{67}$ R. López-Ruiz, H. Mancini, and X. Calbet, "A statistical measure of complexity," Phys. Lett. A 209, 321-326 (1995).

${ }^{68}$ I. Grosse, P. Bernaola-Galván, P. Carpena, R. Román-Roldán, J. Oliver, and H. Stanley, "Analysis of symbolic sequences using the Jensen-Shannon divergence,” Phys. Rev. E 65, 041905 (2002).

${ }^{69}$ M. Zanin, L. Zunino, O. Rosso, and D. Papo, "Permutation entropy and its main biomedical and econophysics applications: A review," Entropy 14, 1553-1577 (2012).

${ }^{70}$ F. Olivares, A. Plastino, and O. Rosso, "Contrasting chaos with noise via local versus global information quantifiers," Phys. Lett. A 376, 1577-1583 (2012).

${ }^{71}$ P. Saco, L. Carpi, A. Figliola, E. Serrano, and O. Rosso, "Entropy analysis of the dynamics of el niño/southern oscillation during the holocene," Phys. A 389, 5022-5027 (2010).

${ }^{72}$ K. Keller and M. Sinn, "Ordinal analysis of time series," Phys. A 356, 114-120 (2005).

${ }^{73}$ L. Zunino, M. Soriano, I. Fischer, O. Rosso, and C. Mirasso, "Permutationinformation-theory approach to unveil delay dynamics from time-series analysis," Phys. Rev. E 82, 046212 (2010).

${ }^{74}$ M. Soriano, L. Zunino, O. Rosso, I. Fischer, and C. Mirasso, "Time scales of a chaotic semiconductor laser with optical feedback under the lens of a permutation information analysis," IEEE J. Quant. Electron. 47, 252-261 (2011).

${ }^{75}$ L. Zunino, M. Soriano, and O. Rosso, "Distinguishing chaotic and stochastic dynamics from time series by using a multiscale symbolic approach," Phys. Rev. E 86, 046210 (2012).

${ }^{76}$ F. Olivares, A. Plastino, and O. Rosso, "Ambiguities in the Bandt-Pompe's methodology for local entropic quantifiers," Phys. A 391, 2518-2526 (2012).

${ }^{77}$ S. Ma, "Calculation of entropy from data of motion," J. Stat. Phys. 26, 221-240 (1981)

${ }^{78}$ S. Strong, R. Koberle, R. de Ruyter van Steveninck, and W. Bialek, "Entropy and information in neural spike trains," Phys. Rev. Lett. 80, 197-200 (1998).

${ }^{79}$ D. Wolpert and D. Wolf, "Estimating functions of probability distributions from a finite set of samples," Phys. Rev. E 52, 6841-54 (1995).

${ }^{80}$ I. Samengo, "Estimating probabilities from experimental frequencies," Phys. Rev. E 65, 046124 (2002).

${ }^{81}$ A. Carlton, "On the bias of information estimates," Psychol. Bull. 71, 108-109 (1969).

${ }^{82}$ S. Panzeri and A. Treves, "Analytical estimates of limited sampling biases in different information measures," Network 7, 87-107 (1996).

${ }^{83}$ S. Panzeri and S. Schultz, "A unified approach to the study of temporal, correlational and rate coding," Neural Comput. 13, 1311-1349 (2001).

${ }^{84}$ N. Rust, S. Schultz, and J. Movshon, "A reciprocal relationship between reliability and responsiveness in developing cortical neurons," J. Neurosci. 22, 10519-10523 (2002).

${ }^{85}$ L. Paninski, "Estimation of entropy and mutual information," Neural Comput. 15, 1191-1254 (2003).

${ }^{86}$ O. Rosso, M. Martín, A. Figliola, K. Keller, and A. Plastino, "Eeg analysis using wavelet-based information tools," J. Neurosci. Methods 153, 163-182 (2006). 